\title{
DEFENSE WASTE PROCESSING FACILITY (DWPF) ANALYTICAL METHOD VERIFICATION FOR THE SLUDGE BATCH 5 QUALIFICATION SAMPLE
}

D. R. Click

H. M. Ajo

T. B. Edwards

July 25, 2008

Analytical Development

Savannah River National Laboratory

Aiken, SC 29808

Prepared for the U.S. Department of Energy Under Contract Number DE-AC09-08SR22470

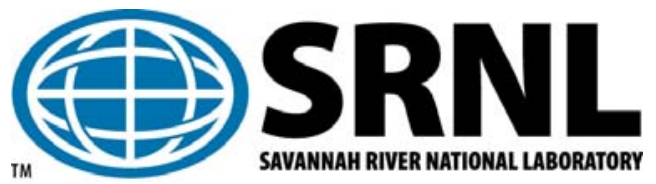


This page intentionally left blank 
DISCLAIMER

This work was prepared under an agreement with and funded by the U.S. Government. Neither the U.S. Government or its employees, nor any of its contractors, subcontractors or their employees, makes any express or implied:

1. warranty or assumes any legal liability for the accuracy, completeness, or for the use or results of such use of any information, product, or process disclosed;

2. representation that such use or results of such use would not infringe privately owned rights; or

3. endorsement or recommendation of any specifically identified commercial product, process, or service. Any views and opinions of authors expressed in this work do not necessarily state or reflect those of the United States Government, or its contractors, or subcontractors.

Printed in the United States of America

Prepared For

U.S. Department of Energy 
This page intentionally left blank 
Key Words:

method development, analytical process

Retention:

Permanent

\title{
DEFENSE WASTE PROCESSING FACILITY (DWPF) ANALYTICAL METHOD VERIFICATION FOR THE SLUDGE BATCH 5 QUALIFICATION SAMPLE
}

\author{
D. R. Click \\ H. M. Ajo
}

T. B. Edwards

July 25, 2008

Analytical Development

Savannah River National Laboratory

Aiken, SC 29808

Prepared for the U.S. Department of Energy Under Contract Number DE-AC09-08SR22470

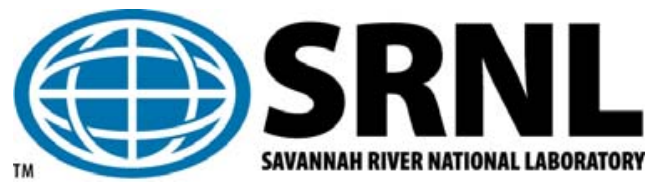




\section{REVIEWS AND APPROVALS}

D.R. Click, Co-Author, Analytical Development, SRNL

Date

H.M. Ajo, Co-Author, Analytical Development, SRNL

Date

T.B. Edwards, Co-Author, Statistical Consulting, SRNL

Date

C.J. Coleman, Technical Reviewer, Analytical Development, SRNL

Date

C.C. Herman, Manager, Process Science Engineering Technology, SRNL

Date

F.M. Pennebaker, Manager, Materials Characterization and Nuclear Measurements, SRNL Date 


\section{TABLE OF CONTENTS}

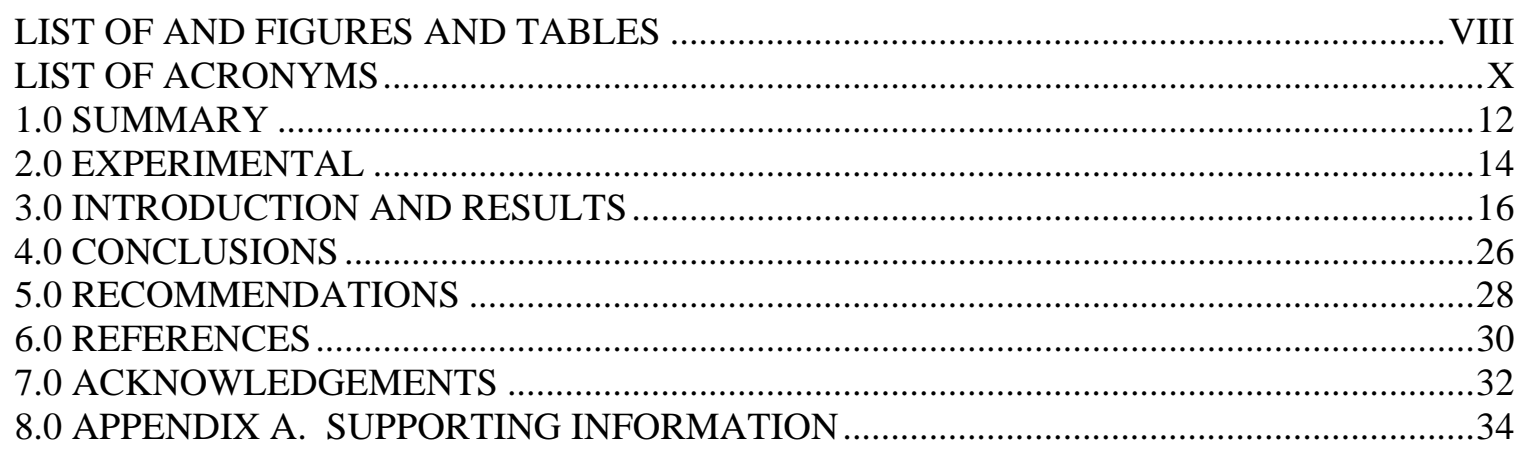


This page intentionally left blank 


\section{LIST OF AND FIGURES AND TABLES}

Figure 3-1. Angle of Intersection and Significance for Compariosn Circles.

Table 3-1. Elemental concentrations of SB5 SRAT Receipt radioactive sludge slurry obtained from ICP-AES analysis of Aqua Regia, DWPF Cold Chem method and Sodium Peroxide/Hydroxide Fusion digestions. Values are presented on a weight percent (Wt\%) total solids basis.

Table 3-2. Statistical comparison of Aqua Regia, DWPF Cold Chem method and Sodium Peroxide/Hydroxide Fusion digestions of SB5 SRAT Receipt sludge. Digestions not having the same letter are statistically different at the $5 \%$ significance level.

Table 3-3. Elemental concentrations of SB5 SRAT Product radioactive sludge slurry obtained from ICP-AES analysis of Aqua Regia, DWPF Cold Chem method and Sodium Peroxide/Hydroxide Fusion digestions. Values are presented on a weight percent (Wt\%) total solids basis.

Table 3-4. Statistical comparison of Aqua Regia, DWPF Cold Chem method and Sodium Peroxide/Hydroxide Fusion digestions of SB5 SRAT Product sludge. Digestions not having the same letter are statistically different at the $5 \%$ significance level.

Table 3-5. Elemental concentrations of ARG standard from ICP-AES analysis of Aqua Regia, DWPF Cold Chem method and Sodium Peroxide/Hydroxide Fusion digestions performed concurrently with SB5 SRAT Receipt. Values are presented on a weight percent (Wt\%) total solids basis.

Table 3-6. Continuation of elemental concentrations of ARG standard from ICP-AES analysis of Aqua Regia, DWPF Cold Chem method and Sodium Peroxide/Hydroxide Fusion digestions performed concurrently with SB5 SRAT Receipt. Values are presented on a weight percent (Wt\%) total solids basis...................23

Table 3-7. Elemental concentrations of ARG standard from ICP-AES analysis of Aqua Regia, DWPF Cold Chem method and Sodium Peroxide/Hydroxide Fusion digestions performed concurrently with SB5 SRAT Product. Values are presented on a weight percent (Wt\%) total solids basis.

Table 3-8. Continuation of elemental concentrations of ARG standard from ICP-AES analysis of Aqua Regia, DWPF Cold Chem method and Sodium Peroxide/Hydroxide Fusion digestions performed concurrently with SB5 SRAT Product. Values are presented on a weight percent (Wt\%) total solids basis. 
This page intentionally left blank 


\section{LIST OF ACRONYMS}

$\mathrm{AD}$

AR

ARG

CC

DI

DOE

DWPF

ICP-ES

PF

PS\&E

SB

SRAT

SRNL
Analytical Development

Aqua Regia

Analytical Reference Glass

Cold Chem Method

De-Ionized

Department of Energy

Defense Waste Processing Facility

Inductively Coupled Plasma Emission Spectroscopy

Sodium Peroxide/Hydroxide Fusion

Process Science and Engineering

Sludge Batch

Slurry Receipt and Adjustment Tank

Savannah River National Laboratory 
This page intentionally left blank 


\subsection{SUMMARY}

For each sludge batch that is processed in the Defense Waste Processing Facility (DWPF), the Savannah River National Laboratory (SRNL) performs confirmation of the applicability of the digestion method to be used by the DWPF lab for elemental analysis of Sludge Receipt and Adjustment Tank (SRAT) receipt samples and SRAT product process control samples. DWPF SRAT samples are typically dissolved using a room temperature $\mathrm{HF}-\mathrm{HNO}_{3}$ acid dissolution (i.e., DWPF Cold Chem Method, see Procedure SW415.201) and then analyzed by inductively coupled plasma - atomic emission spectroscopy (ICP-AES).

This report contains the results and comparison of data generated from performing the Aqua Regia (AR), Sodium Peroxide/Hydroxide Fusion (PF) and DWPF Cold Chem (CC) method digestion of Sludge Batch 5 (SB5) SRAT Receipt and SB5 SRAT Product samples. The SB5 SRAT Receipt and SB5 SRAT Product samples were prepared in the SRNL Shielded Cells, and the SRAT Receipt material is representative of the sludge that constitutes the SB5 Batch composition. This is the sludge in Tank 51 that is to be transferred into Tank 40, which will contain the heel of Sludge Batch 4 (SB4), to form the SB5 Blend composition. The results for any one particular element should not be used in any way to identify the form or speciation of a particular element in the sludge or used to estimate ratios of compounds in the sludge.

A statistical comparison of the data validates the use of the DWPF CC method for SB5 Batch composition. However, the difficulty that was encountered in using the CC method for SB4 brings into question the adequacy of CC for the SB5 Blend. Also, it should be noted that visible solids remained in the final diluted solutions of all samples digested by this method at SRNL (8 samples total), which is typical for the DWPF CC method but not seen in the other methods.

Recommendations to the DWPF for application to SB5 based on studies to date:

- A dissolution study should be performed on the WAPS sample by SRNL which consists of the final composition of the sludge (the SB5 Blend).

- Given the heel of SB4 in Tank 40, the DWPF lab should monitor the aluminum concentration in the first 10 SRAT Receipt batches of SB5 using both CC and sodium peroxide/hydroxide fusion to evaluate the adequacy of aluminum recovery by the CC method for this sludge batch.

- SRNL and the DWPF lab should investigate if comparisons between the elemental concentrations of the SME product glass (adjusted for frit addition) obtained by the mixed acid and peroxide fusion digestion and the SRAT Receipt and SRAT Product elemental concentrations obtained via the DWPF CC method provide insight into the adequacy of the CC method for analysis of the SRAT Product. The DWPF lab would need to calcine the SRAT product at $1050{ }^{\circ} \mathrm{C}$ for the best comparison. If a consistent difference in elemental concentrations is revealed, another type of digestion (i.e. sodium peroxide/hydroxide fusion) should be used to determine the concentration of the element in question. Particular emphasis should be placed on monitoring the aluminum concentration in SB5. 
This page intentionally left blank 


\subsection{EXPERIMENTAL}

The radioactive sludge slurry used in this study for verification of the DWPF CC method is from the three liter sample of Tank 51 sludge slurry taken on March 21, 2008. The sample was delivered to SRNL, characterized and then modified by a series of wash/decant cycles to match the LWO planned preparation strategy. The sludge used in this testing corresponded to Wash F, identified by Bannochie et. al. in SRNL-PSE-2008-1126, which is the SB5 qualification sample.

The sludge samples were dissolved in quadruplicate in the SRNL Shielded Cells facility in a manner similar to the DWPF CC method, and by PF and AR digestion. For detailed steps of the PF digestion, see ADS procedure 2227. ${ }^{1}$ For detailed steps of the AR digestion, see ADS procedure 2502. ${ }^{2}$ Three replicate dissolutions of the analytical reference glass (ARG) standard were performed concurrently with each set of digestions above. Additional quality control measures included ICP-AES analyses of a multi-element standard as a check for ICP-AES accuracy independent of digestions.

The CC method digestion involved adding $25 \mathrm{~mL}$ of concentrated HF to radioactive sludge slurry ( $\sim 6.0 \mathrm{~g}$ for the SRAT Receipt at $17.1 \mathrm{wt} \%$ total solids and $\sim 4.0 \mathrm{~g}$ for the SRAT Product at $26.52 \mathrm{wt} \%$ total solids) and stirring for $1 \mathrm{hr}$. Then, $25 \mathrm{~mL}$ of concentrated $\mathrm{HNO}_{3}$ was added and the mixture was stirred for an additional 30 minutes prior to diluting with de-ionized (DI) water to $250 \mathrm{~mL}$ in a pre-weighed volumetric flask. The density of the solution was obtained from the weight of the $250 \mathrm{~mL}$ of solution.

Approximately $5 \mathrm{~mL}$ was taken from the $250 \mathrm{~mL}$ volumetric flask and added to a pre-weighed $100 \mathrm{~mL}$ volumetric flask. The exact volume of the solution diluted was obtained from the weight of the solution and its density. The effective dilution was $\sim 5000$ fold. Visible solids remained in each radioactive sample bottle. During digestion of the SRAT product, the weight of the solution transferred to the 100 $\mathrm{mL}$ volumetric flasks inadvertently was not recorded, and, therefore, the exact volume of solution transferred could not be calculated. Therefore, the average mass of solution transferred to the $100 \mathrm{~mL}$ volumetric flask during digestion of the SRAT Receipt was used as the mass transferred for the SRAT Product solution. These values were subsequently used to calculate the total digestion factor for the SRAT Product samples. Approximately $0.25 \mathrm{~g}$ of ARG powdered glass were dissolved in triplicate and serial diluted $\sim 5000$ fold with de-ionized water with each set of digestions. 
This page intentionally left blank 


\subsection{INTRODUCTION AND RESULTS}

The DWPF is currently processing and immobilizing radioactive sludge slurry into a durable borosilicate glass. The DWPF has already processed four sludge batches (Sludge Batch 1A, Sludge Batch 1B, Sludge Batch 2 and Sludge Batch 3) and is currently processing a fifth (Sludge Batch 4). A sludge batch is defined as a single tank of sludge or a combination of sludges from different tanks that has been or will be qualified before being transferred to DWPF. Thus, following the sludge batch preparation plan of the Liquid Waste Organization (LWO), the qualified sludge in Tank 51 is to be blended with the heel of the previous sludge batch in Tank 40. The sludge being qualified at the SRNL (referred to as a "batch" composition in sludge batch planning) is to be combined with the heel of the previous sludge batch in DWPF to yield the "blend" composition. The next batch of radioactive sludge slurry to be processed by the DWPF is SB5. The subject of this report is the SB5 material from Tank 51 that was qualified at SRNL, which is the SB5 Batch sludge slurry, while the SB5 Blend will consist of the following:

Sludge Batch 4 heel

Sludge slurry from Tank 7

$\mathrm{Pu} / \mathrm{Np}$ solution from $\mathrm{H}$-canyon

LWO performed a caustic wash of the Sludge Batch 4 slurry remaining in Tank 51 to reduce the aluminum concentration and the total mass of sludge solids being fed to the DWPF before combining with the other materials. ${ }^{3}$

The radioactive sludge slurry used in this study for verification of the DWPF CC method is from the three liter sample of Tank 51 sludge slurry taken on March 21, 2008. The sample was delivered to SRNL, characterized and then modified by a series of wash/decant cycles to match the LWO planned preparation strategy. The sludge used in this testing corresponded to Wash F, identified by Bannochie et. al. in SRNL-PSE-2008-1126, which is the SB5 qualification sample.

The SRAT receipt and SRAT product analytical sub-samples were digested in quadruplicate using the AR, PF and the DWPF CC method. Three ARG samples were digested concurrently with each set of digestions and two multi-element ICP-AES standards were submitted along with each sample batch for analysis containing known concentrations of $\mathrm{Al}, \mathrm{B}, \mathrm{Fe}, \mathrm{Li}, \mathrm{Na}$, and $\mathrm{Si}$.

The measurements of the SB5 Batch samples generated from this study are provided in Table A1 of Appendix A. The results from each type of digestion are summarized in Table 3-1 for the SRAT Receipt and Table 3-3 for the SRAT Product samples. The ICP-AES results of the sixteen elements that are analyzed by the DWPF lab are presented on a weight percent (Wt\%) of total solids basis. Sodium (Na) and zirconium (Zr) are not reported for the PF digestions, which are performed in a Zr crucible using $\mathrm{Na}$ containing reagents. Silicon (Si) is not reported for the DWPF CC method because HF in the solution leaches Si out the nebulizer of the ICP-AES instrument at SRNL.

Statistical comparisons of the data from the three digestion methods (for Na there are only two digestion methods) are provided in Exhibit A1 in Appendix A. The results were generated using JMP Version 6.0.3. ${ }^{4}$ Note that $\mathrm{Zr}$ is shown for the PF digestions and $\mathrm{Si}$ is shown for the CC digestions. The plots of this exhibit show a 95\% confidence interval for the mean (a mean diamond) of each set of measurements as well as comparison circles for the digestion means.

Comparisons of each pair of digestion means for each element except Na can be made visually by examining how the comparison circles intersect. The outside angle of intersection conveys whether the digestion means are significantly different (Figure 3-1). Circles for means that are significantly different 
either do not intersect or intersect slightly so that the outside angle of intersection is less than 90 degrees. If the circles intersect by an angle of more than 90 degrees or if they are nested, the means are not significantly different (at an overall $5 \%$ level of significance). ${ }^{4}$

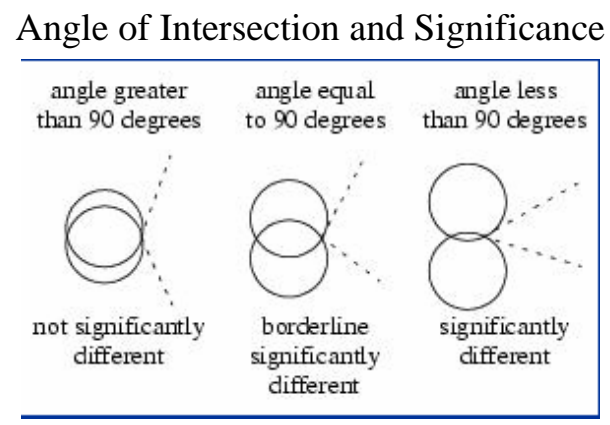

Figure 3-1. Angle of Intersection and Significance for Compariosn Circles.

While there may be difficulty in interpreting the comparison circles for some of the elements, the exhibit also contains some tabulated results to help in drawing conclusions from these results. For each element, the mean concentration of the samples by each digestion method is provided, and means that are not connected by the same letter in the listing of the exhibit are significantly different. For example consider the SRAT Product Al measurements. The results of the exhibit indicate that the mean of the AR results differs from the mean of the PF and CC results.

For the $\mathrm{Na}, \mathrm{Si}$, and $\mathrm{Zr}$ comparisons, the JMP output from an analysis of variance of the measurements for two digestions is provided, and only the 95\% confidence mean diamond of each digestion is shown. Overlap marks show for each diamond, and overlap marks in one diamond that are closer to the mean of another diamond than that diamond's overlap marks indicate that those two groups are not different at the 95\% confidence level. The visual comparisons are supported by an F test that compares the means of the $\mathrm{AR}$ and $\mathrm{CC}$ digestions for $\mathrm{Na}$ and $\mathrm{Zr}$ and the means of the $\mathrm{AR}$ and $\mathrm{PF}$ digestions for $\mathrm{Si}$. If the $\mathrm{p}$ value is less than 0.05 , then the means are statistically different at the $5 \%$ level. From Exhibit A1, there is an indication of a difference in the AR and CC means for the SRAT Product for Na but not the SRAT Receipt Na.

Summaries of the statistical comparisons of Exhibit A1 are shown in Table 3-2 and Table 3-4. Following the format used in the exhibit, digestions not having the same letter are statistically different at the 5\% significance level. Consider the SRAT Receipt Al results, the average mean of Al measured in the PF and DWPF CC method digestions are statistically the same (and both columns have the letter A), but these means are statistically different from the mean average obtained from the AR digestion (which has the letter B in the column). ARG results are presented and compared for each digestion type for the SRAT Receipt and SRAT Product samples in Tables 5 and 6, respectively.

Undissolved solids remained in the DWPF Cold Chem digestate solutions in each case. The identity of the undissolved solids has not been determined for these samples. However, during verification of the DWPF CC method for previous sludge batches, boehmite (AlO(OH)), muscovite (K,Na)(Al, Mg, $\mathrm{Fe})_{2}\left(\mathrm{Si}_{3.1} \mathrm{Al}_{0.9}\right) \mathrm{O}_{10}(\mathrm{OH})_{2}$, silicon dioxide $\left(\mathrm{SiO}_{2}\right)$, potassium sodium aluminum fluoride $\left(\mathrm{K}_{2} \mathrm{NaAl}_{3} \mathrm{~F}_{12}\right)$, potassium aluminum fluoride $\left(\mathrm{K}_{2} \mathrm{AlF}_{5}\right)$, aluminum fluoride $\left(\mathrm{AlF}_{3}\right)$, chiolite $\left(\mathrm{Na}_{5} \mathrm{Al}_{3} \mathrm{~F}_{14}\right)$, cryolite $\left(\mathrm{Na}_{3} \mathrm{AlF}_{6}\right)$, sodium magnesium aluminum hexafluoride $\left(\mathrm{NaMgAlF}_{6}\right), \mathrm{FeZrF}_{6}$ and $\mathrm{Na}_{2} \mathrm{FeAlF}_{7}$ have been found. ${ }^{5}$ No undissolved solids were noticed in the PF or AR solutions by visual inspection. 
Table 3-1. Elemental concentrations of SB5 SRAT Receipt radioactive sludge slurry obtained from ICP-AES analysis of Aqua Regia, DWPF Cold Chem method and Sodium Peroxide/Hydroxide Fusion digestions. Values are presented on a weight percent $(\mathrm{Wt} \%)$ total solids basis.

\begin{tabular}{|c|c|c|c|c|c|c|}
\hline Element & $\begin{array}{c}\text { Aqua Regia } \\
\text { Digestion } \\
\text { Avg Wt\%* }\end{array}$ & \%RSD & $\begin{array}{c}\mathrm{Na}_{2} \mathrm{O}_{2} / \\
\mathrm{NaOH} \\
\text { Fusion } \\
\text { Digestion } \\
\text { Avg Wt\%* }\end{array}$ & \%RSD & $\begin{array}{c}\text { DWPF Cold } \\
\text { Chem } \\
\text { Digestion } \\
\text { Avg Wt\%* }\end{array}$ & \%RSD \\
\hline $\mathrm{Al}$ & $7.62 \mathrm{E}+00$ & $6.4 \mathrm{E}+00$ & $8.91 \mathrm{E}+00$ & $1.9 \mathrm{E}+00$ & $8.87 \mathrm{E}+00$ & $4.9 \mathrm{E}+00$ \\
\hline $\mathrm{B}$ & $<2.63 \mathrm{E}-02$ & NA & $<2.62 \mathrm{E}-02$ & NA & $<1.33 \mathrm{E}-01$ & NA \\
\hline $\mathrm{Ca}$ & $1.31 \mathrm{E}+00$ & $1.9 \mathrm{E}+00$ & $1.34 \mathrm{E}+00$ & $2.9 \mathrm{E}+00$ & $1.35 \mathrm{E}+00$ & $1.9 \mathrm{E}+00$ \\
\hline $\mathrm{Cr}$ & $4.67 \mathrm{E}-02$ & $3.1 \mathrm{E}+00$ & $1.41 \mathrm{E}-01$ & $1.1 \mathrm{E}+02$ & $4.24 \mathrm{E}-02$ & $5.9 \mathrm{E}-01$ \\
\hline $\mathrm{Cu}$ & $6.45 \mathrm{E}-02$ & $3.3 \mathrm{E}+00$ & $6.02 \mathrm{E}-02$ & $2.9 \mathrm{E}+00$ & $5.75 \mathrm{E}-02$ & $1.3 \mathrm{E}+00$ \\
\hline $\mathrm{Fe}$ & $1.60 \mathrm{E}+01$ & $2.1 \mathrm{E}+00$ & $1.65 \mathrm{E}+01$ & $4.6 \mathrm{E}+00$ & $1.53 \mathrm{E}+01$ & $2.0 \mathrm{E}+00$ \\
\hline $\mathrm{Li}$ & $3.54 \mathrm{E}-02$ & $5.6 \mathrm{E}+00$ & $3.62 \mathrm{E}-02$ & $6.4 \mathrm{E}+00$ & $3.11 \mathrm{E}-02$ & $1.1 \mathrm{E}+01$ \\
\hline $\mathrm{K}$ & $<1.18 \mathrm{E}-01$ & NA & $7.85 \mathrm{E}-01$ & $7.1 \mathrm{E}+00$ & $<6.00 \mathrm{E}-01$ & NA \\
\hline $\mathrm{Mg}$ & $6.81 \mathrm{E}-01$ & $1.9 \mathrm{E}+00$ & $6.04 \mathrm{E}-01$ & $6.4 \mathrm{E}-01$ & $6.09 \mathrm{E}-01$ & $1.6 \mathrm{E}+00$ \\
\hline $\mathrm{Mn}$ & $3.74 \mathrm{E}+00$ & $1.9 \mathrm{E}+00$ & $3.58 \mathrm{E}+00$ & $1.2 \mathrm{E}+00$ & $3.61 \mathrm{E}+00$ & $1.3 \mathrm{E}+00$ \\
\hline $\mathrm{Na}$ & $1.52 \mathrm{E}+01$ & $1.7 \mathrm{E}+00$ & NA & NA & $1.54 \mathrm{E}+01$ & $1.4 \mathrm{E}+00$ \\
\hline $\mathrm{Ni}$ & $2.32 \mathrm{E}+00$ & $1.2 \mathrm{E}+00$ & $2.37 \mathrm{E}+00$ & $2.4 \mathrm{E}+00$ & $2.24 \mathrm{E}+00$ & $1.7 \mathrm{E}+00$ \\
\hline $\mathrm{Si}$ & $3.97 \mathrm{E}-01$ & $3.4 \mathrm{E}+01$ & $9.23 \mathrm{E}-01$ & $1.6 \mathrm{E}+00$ & NA & NA \\
\hline $\mathrm{Ti}$ & $1.96 \mathrm{E}-02$ & $2.7 \mathrm{E}+00$ & $2.03 \mathrm{E}-02$ & $1.5 \mathrm{E}+00$ & $1.99 \mathrm{E}-02$ & $7.1 \mathrm{E}+00$ \\
\hline $\mathrm{U}$ & $5.58 \mathrm{E}+00$ & $1.7 \mathrm{E}+00$ & $5.57 \mathrm{E}+00$ & $8.2 \mathrm{E}-01$ & $5.67 \mathrm{E}+00$ & $1.5 \mathrm{E}+00$ \\
\hline $\mathrm{Zr}$ & $9.16 \mathrm{E}-02$ & $6.2 \mathrm{E}+01$ & NA & NA & $2.59 \mathrm{E}-01$ & $1.3 \mathrm{E}+00$ \\
\hline
\end{tabular}

*All averages are based upon four replicate dissolutions and ICP-AES determinations. NA = Not Applicable. ${ }^{\#} \%$ RSD is the percent relative standard deviation for the measurements. 
Table 3-2. Statistical comparison of Aqua Regia, DWPF Cold Chem method and Sodium Peroxide/Hydroxide Fusion digestions of SB5 SRAT Receipt sludge. Digestions not having the same letter are statistically different at the $5 \%$ significance level.

\begin{tabular}{|c|c|c|c|}
\hline Element & $\begin{array}{c}\text { Aqua } \\
\text { Regia } \\
\text { Digestion* }\end{array}$ & $\begin{array}{c}\mathrm{Na}_{2} \mathrm{O}_{2} / \\
\mathrm{NaOH} \\
\text { Fusion } \\
\text { Digestion* }\end{array}$ & $\begin{array}{c}\text { DWPF Cold } \\
\text { Chem } \\
\text { Method } \\
\text { Digestion* }\end{array}$ \\
\hline $\mathrm{Al}$ & $\mathrm{B}$ & $\mathrm{A}$ & $\mathrm{A}$ \\
\hline $\mathrm{B}$ & $<\mathrm{MDL}$ & $<\mathrm{MDL}$ & $<$ MDL \\
\hline $\mathrm{Ca}$ & $\mathrm{A}$ & $\mathrm{A}$ & $\mathrm{A}$ \\
\hline $\mathrm{Cr}$ & $\mathrm{A}$ & $\mathrm{A}$ & $\mathrm{A}$ \\
\hline $\mathrm{Cu}$ & $\mathrm{A}$ & $\mathrm{B}$ & $\mathrm{B}$ \\
\hline $\mathrm{Fe}$ & $\mathrm{AB}$ & $\mathrm{A}$ & $\mathrm{B}$ \\
\hline $\mathrm{K}$ & $<\mathrm{MDL}$ & $\mathrm{A}$ & $<\mathrm{MDL}$ \\
\hline $\mathrm{Li}$ & $\mathrm{A}$ & $\mathrm{A}$ & $\mathrm{A}$ \\
\hline $\mathrm{Mg}$ & $\mathrm{A}$ & $\mathrm{B}$ & $\mathrm{B}$ \\
\hline $\mathrm{Mn}$ & $\mathrm{A}$ & $\mathrm{B}$ & $\mathrm{B}$ \\
\hline $\mathrm{Na}$ & $\mathrm{A}$ & $\mathrm{NA}$ & $\mathrm{B}$ \\
\hline $\mathrm{Ni}$ & $\mathrm{AB}$ & $\mathrm{A}$ & $\mathrm{B}$ \\
\hline $\mathrm{Si}$ & $\mathrm{B}$ & $\mathrm{B}$ & $\mathrm{NA}$ \\
\hline $\mathrm{Ti}$ & $\mathrm{A}$ & $\mathrm{A}$ & $\mathrm{A}$ \\
\hline $\mathrm{U}$ & $\mathrm{A}$ & $\mathrm{A}$ & $\mathrm{A}$ \\
\hline $\mathrm{Zr}$ & $\mathrm{B}$ & $\mathrm{NA}$ & $\mathrm{A}$ \\
\hline
\end{tabular}

*All averages are based upon four replicate dissolutions and ICP-AES determinations. NA = Not Applicable. $<$ MDL = Less than minimum detection limit.

The SRAT Receipt sample digested by the PF and DWPF CC methods have a statistical difference in the means for Fe and Ni out of the major elements ( $>1.0 \mathrm{wt} \%$ total solids basis - Al, Ca, Fe, Mn, Ni and U). The relative difference between the mean concentration for Fe in the DWPF CC digestions and the PF digestions is $7.6 \%$. The relative difference for $\mathrm{Ni}$ is $5.2 \%$.

The SRAT Receipt sample digested by the AR and DWPF CC methods have a statistical difference in the means for $\mathrm{Al}$ and $\mathrm{Mn}$ out of the major elements (>1.0 wt\% total solids basis - $\mathrm{Al}, \mathrm{Ca}, \mathrm{Fe}, \mathrm{Mn}, \mathrm{Ni}$ and $\mathrm{U}$ ). The relative difference between the mean results for $\mathrm{Al}$ in the DWPF CC method digestions and the AR digestions is $15 \%$. The relative difference for $\mathrm{Mn}$ is $3.4 \%$.

A statistical difference in the mean result for $\mathrm{Al}$ and $\mathrm{Mn}$ is noted for the SRAT Receipt sample digested by the AR and PF methods. The relative difference between the mean results for $\mathrm{Al}$ in the AR digestions and the PF digestions is $16 \%$. The relative difference for $\mathrm{Mn}$ is $4.2 \%$.

The statistical difference noted for elements having a relative small difference (Fe, $\mathrm{Ni}$ and $\mathrm{Mn}$ ) indicate a tight precision in the ICP-AES measurements. It is unclear why the aluminum value is so low in the AR digestion. As noted above, there were no undissolved solids in the final diluted solutions. The $\mathrm{Al}$ concentration obtained by AR digestion of the ARG was high by $\sim 1 \%$ relative to the standard value for this reference glass. The Al value obtained by the DWPF CC method for the ARG glass was $\sim 5 \%$ high relative to the standard value and the Al value was high by $\sim 1 \%$ for the ARG digested by the PF method. Note that PF and CC Al concentrations are statistically equivalent for both the SB5 SRAT Receipt and SRAT Product sample given the disparity for SB4. ${ }^{6}$ 
Table 3-3. Elemental concentrations of SB5 SRAT Product radioactive sludge slurry obtained from ICP-AES analysis of Aqua Regia, DWPF Cold Chem method and Sodium Peroxide/Hydroxide Fusion digestions. Values are presented on a weight percent $(\mathrm{Wt} \%)$ total solids basis.

\begin{tabular}{|c|c|c|c|c|c|c|}
\hline Element & $\begin{array}{l}\text { Aqua Regia } \\
\text { Digestion } \\
\text { Avg } \mathrm{Wt} \% *\end{array}$ & $\% \mathrm{RSD}^{\#}$ & \begin{tabular}{|l|}
$\mathrm{Na}_{2} \mathrm{O}_{2} /$ \\
$\mathrm{NaOH}$ \\
Fusion \\
Digestion \\
Avg Wt\%* \\
\end{tabular} & $\%$ RSD & $\begin{array}{l}\text { DWPF Cold } \\
\text { Chem } \\
\text { Digestion } \\
\text { Avg Wt\%* }\end{array}$ & $\% \mathrm{RSD}^{\#}$ \\
\hline $\mathrm{Al}$ & $5.37 \mathrm{E}+00$ & $3.6 \mathrm{E}+00$ & $7.14 \mathrm{E}+00$ & $2.9 \mathrm{E}+00$ & $6.70 \mathrm{E}+00$ & $4.0 \mathrm{E}+00$ \\
\hline $\mathrm{B}$ & $<2.41 \mathrm{E}-02$ & NA & $<2.34 \mathrm{E}-02$ & NA & 1.99E-01 & $1.6 \mathrm{E}+01$ \\
\hline $\mathrm{Ca}$ & $1.13 \mathrm{E}+00$ & $3.2 \mathrm{E}+00$ & $1.07 \mathrm{E}+00$ & $3.6 \mathrm{E}+00$ & $1.07 \mathrm{E}+00$ & $2.3 \mathrm{E}+00$ \\
\hline $\mathrm{Cr}$ & 3.95E-02 & $3.2 \mathrm{E}+00$ & 3.99E-02 & $7.0 \mathrm{E}+00$ & 3.97E-02 & $2.9 \mathrm{E}+00$ \\
\hline $\mathrm{Cu}$ & 5.24E-02 & $4.9 \mathrm{E}+00$ & 4.64E-02 & $4.5 \mathrm{E}+00$ & 4.76E-02 & $4.5 \mathrm{E}+00$ \\
\hline $\mathrm{Fe}$ & $1.35 \mathrm{E}+01$ & $3.5 \mathrm{E}+00$ & $1.24 \mathrm{E}+01$ & $3.2 \mathrm{E}+00$ & $1.30 \mathrm{E}+01$ & $2.0 \mathrm{E}+00$ \\
\hline $\mathrm{Li}$ & 2.86E-02 & $3.4 \mathrm{E}+00$ & 3.18E-02 & 7.3E+00 & 2.89E-02 & $2.6 \mathrm{E}+00$ \\
\hline $\mathrm{K}$ & $<1.08 \mathrm{E}-01$ & NA & $<5.27 \mathrm{E}-01$ & NA & $<5.65 \mathrm{E}-01$ & NA \\
\hline $\mathrm{Mg}$ & 6.04E-01 & $3.0 \mathrm{E}+00$ & 5.25E-01 & $3.3 \mathrm{E}+00$ & 5.51E-01 & $1.5 \mathrm{E}+00$ \\
\hline Mn & $3.15 \mathrm{E}+00$ & $3.5 \mathrm{E}+00$ & $3.02 \mathrm{E}+00$ & $3.4 \mathrm{E}+00$ & $3.21 \mathrm{E}+00$ & $1.4 \mathrm{E}+00$ \\
\hline $\mathrm{Na}$ & $1.28 \mathrm{E}+01$ & $3.4 \mathrm{E}+00$ & NA & NA & $1.36 \mathrm{E}+01$ & $2.4 \mathrm{E}+00$ \\
\hline $\mathrm{Ni}$ & $2.05 \mathrm{E}+00$ & $3.4 \mathrm{E}+00$ & $1.94 \mathrm{E}+00$ & $3.1 \mathrm{E}+00$ & $2.01 \mathrm{E}+00$ & $1.9 \mathrm{E}+00$ \\
\hline Si & 3.44E-01 & $1.9 \mathrm{E}+01$ & 7.57E-01 & $5.5 \mathrm{E}+00$ & NA & NA \\
\hline $\mathrm{Ti}$ & 1.38E-02 & $3.3 \mathrm{E}+00$ & 1.71E-02 & $4.0 \mathrm{E}+00$ & 1.58E-02 & $2.2 \mathrm{E}+00$ \\
\hline $\mathrm{U}$ & $4.82 \mathrm{E}+00$ & $3.9 \mathrm{E}+00$ & $4.94 \mathrm{E}+00$ & $2.8 \mathrm{E}+00$ & $4.87 \mathrm{E}+00$ & 2.9E-01 \\
\hline $\mathrm{Zr}$ & 9.85E-02 & $9.6 \mathrm{E}+00$ & NA & NA & 2.19E-01 & $1.1 \mathrm{E}+00$ \\
\hline
\end{tabular}

*All averages are based upon four replicate dissolutions and ICP-AES determinations. $\mathrm{NA}=$ Not Applicable. ${ }^{\#} \%$ RSD is the percent relative standard deviation for the measurements. 
Table 3-4. Statistical comparison of Aqua Regia, DWPF Cold Chem method and Sodium Peroxide/Hydroxide Fusion digestions of SB5 SRAT Product sludge. Digestions not having the same letter are statistically different at the $5 \%$ significance level.

\begin{tabular}{|c|c|c|c|}
\hline Element & $\begin{array}{c}\text { Aqua } \\
\text { Regia } \\
\text { Digestion** }\end{array}$ & $\begin{array}{c}\mathrm{Na}_{2} \mathrm{O}_{2} / \\
\mathrm{NaOH} \\
\text { Fusion } \\
\text { Digestion* }\end{array}$ & $\begin{array}{c}\text { DWPF Cold } \\
\text { Chem } \\
\text { Method } \\
\text { Digestion* }\end{array}$ \\
\hline $\mathrm{Al}$ & $\mathrm{B}$ & $\mathrm{A}$ & $\mathrm{A}$ \\
\hline $\mathrm{B}$ & $<\mathrm{MDL}$ & $<\mathrm{MDL}$ & $\mathrm{A}$ \\
\hline $\mathrm{Ca}$ & $\mathrm{A}$ & $\mathrm{A}$ & $\mathrm{A}$ \\
\hline $\mathrm{Cr}$ & $\mathrm{A}$ & $\mathrm{A}$ & $\mathrm{A}$ \\
\hline $\mathrm{Cu}$ & $\mathrm{A}$ & $\mathrm{B}$ & $\mathrm{B}$ \\
\hline $\mathrm{Fe}$ & $\mathrm{A}$ & $\mathrm{B}$ & $\mathrm{AB}$ \\
\hline $\mathrm{K}$ & $<\mathrm{MDL}$ & $<\mathrm{MDL}$ & $<\mathrm{MDL}$ \\
\hline $\mathrm{Li}$ & $\mathrm{B}$ & $\mathrm{A}$ & $\mathrm{AB}$ \\
\hline $\mathrm{Mg}$ & $\mathrm{A}$ & $\mathrm{B}$ & $\mathrm{B}$ \\
\hline $\mathrm{Mn}$ & $\mathrm{AB}$ & $\mathrm{B}$ & $\mathrm{A}$ \\
\hline $\mathrm{Na}$ & $\mathrm{A}$ & $\mathrm{NA}$ & $\mathrm{A}$ \\
\hline $\mathrm{Ni}$ & $\mathrm{A}$ & $\mathrm{A}$ & $\mathrm{A}$ \\
\hline $\mathrm{Si}$ & $\mathrm{B}$ & $\mathrm{B}$ & $\mathrm{NA}$ \\
\hline $\mathrm{Ti}$ & $\mathrm{C}$ & $\mathrm{A}$ & $\mathrm{B}$ \\
\hline $\mathrm{U}$ & $\mathrm{A}$ & $\mathrm{A}$ & $\mathrm{A}$ \\
\hline $\mathrm{Zr}$ & $\mathrm{B}$ & $\mathrm{NA}$ & $\mathrm{A}$ \\
\hline
\end{tabular}

*All averages are based upon four replicate dissolutions and ICP-AES determinations. NA $=$ Not Applicable. $<$ MDL $=$ Less than minimum detection limit.

The SRAT Product sample digested by the PF and DWPF CC methods have a statistical difference in the means for Mn out of the major elements ( $>1.0 \mathrm{wt} \%$ total solids basis - $\mathrm{Al}, \mathrm{Ca}, \mathrm{Fe}, \mathrm{Mn}, \mathrm{Ni}$ and $\mathrm{U})$. The relative difference between the mean concentration for Mn in the DWPF CC digestions and the PF digestions is $6.3 \%$.

The SRAT Product sample digested by the AR and DWPF CC methods have a statistical difference in the means for $\mathrm{Al}$ and $\mathrm{Na}$ out of the major elements (>1.0 wt\% total solids basis - $\mathrm{Al}, \mathrm{Ca}, \mathrm{Fe}, \mathrm{Mn}, \mathrm{Ni}$ and $\mathrm{U}$ ). The relative difference between the mean results for Al in the DWPF CC method digestions and the AR digestions is $22 \%$, and the relative difference between the mean results for $\mathrm{Na}$ in the DWPF CC method digestions and the AR digestions is $6.1 \%$.

A statistical difference in the mean result for $\mathrm{Al}$ and Fe is noted for the SRAT Product sample digested by the AR and PF methods. The relative difference between the mean results for $\mathrm{Al}$ in the AR digestions and the $\mathrm{PF}$ digestions is $28 \%$. The relative difference for $\mathrm{Fe}$ is $8.5 \%$.

Table 3-5, Table 3-6, Table 3-7, and Table 3-8 contain the ICP-AES measured weight percent elemental results from triplicate digestions of the ARG standard performed concurrently with the SB5 radioactive sludge to determine if the dissolutions were complete and the resulting analyses accurate. Comparison to the known elemental weight percent in the ARG standard is also given in Tables 5-8. The experimentally measured values agree well with the ARG standards for all three digestions where expected. 
Table 3-5. Elemental concentrations of ARG standard from ICP-AES analysis of Aqua Regia, DWPF Cold Chem method and Sodium Peroxide/Hydroxide Fusion digestions performed concurrently with SB5 SRAT Receipt. Values are presented on a weight percent $(\mathrm{Wt} \%)$ total solids basis.

\begin{tabular}{|c|c|c|c|c|}
\hline \multicolumn{5}{|l|}{ Aqua Regia* } \\
\hline Element & Average & \%RSD & Standard Value & $\begin{array}{c}\text { \%Difference } \\
\text { (Measured vs } \\
\text { Standard Value) }\end{array}$ \\
\hline $\mathrm{Al}$ & $2.52 \mathrm{E}+00$ & $2.6 \mathrm{E}+00$ & $2.50 \mathrm{E}+00$ & 0.6 \\
\hline B & $2.77 \mathrm{E}+00$ & $3.3 \mathrm{E}+00$ & $2.69 \mathrm{E}+00$ & 3.0 \\
\hline $\mathrm{Ca}$ & $1.12 \mathrm{E}+00$ & $2.3 \mathrm{E}+00$ & $1.02 \mathrm{E}+00$ & 9.3 \\
\hline $\mathrm{Cr}$ & 7.02E-02 & $2.9 \mathrm{E}+00$ & $6.40 \mathrm{E}-02$ & 9.7 \\
\hline $\mathrm{Cu}$ & $<1.00 \mathrm{E}-02$ & NA & $3.00 \mathrm{E}-03$ & NA \\
\hline $\mathrm{Fe}$ & $1.04 \mathrm{E}+01$ & $2.7 \mathrm{E}+00$ & $9.79 \mathrm{E}+00$ & 5.7 \\
\hline $\mathrm{K}$ & $2.34 \mathrm{E}+00$ & $1.5 \mathrm{E}+00$ & $2.26 \mathrm{E}+00$ & 3.5 \\
\hline $\mathrm{Li}$ & $1.55 \mathrm{E}+00$ & $2.6 \mathrm{E}+00$ & $1.49 \mathrm{E}+00$ & 4.0 \\
\hline $\mathrm{Mg}$ & 6.07E-01 & $2.7 \mathrm{E}+00$ & $5.20 \mathrm{E}-01$ & 16.6 \\
\hline $\mathrm{Mn}$ & $1.50 \mathrm{E}+00$ & $3.0 \mathrm{E}+00$ & $1.46 \mathrm{E}+00$ & 2.4 \\
\hline $\mathrm{Na}$ & $9.04 \mathrm{E}+00$ & $2.7 \mathrm{E}+00$ & $8.52 \mathrm{E}+00$ & 6.0 \\
\hline $\mathrm{Ni}$ & 8.42E-01 & $3.6 \mathrm{E}+00$ & 8.27E-01 & 1.8 \\
\hline $\mathrm{Si}$ & 4.15E-01 & $4.8 \mathrm{E}+01$ & $2.24 \mathrm{E}+01$ & NA \\
\hline $\mathrm{Ti}$ & 5.97E-01 & $5.0 \mathrm{E}+00$ & $6.90 \mathrm{E}-01$ & $\begin{array}{l}-13.6 \\
\end{array}$ \\
\hline U & NA & NA & NA & NA \\
\hline $\mathrm{Zr}$ & $5.16 \mathrm{E}-02$ & $4.3 \mathrm{E}+01$ & $9.60 \mathrm{E}-02$ & -46.3 \\
\hline \multicolumn{5}{|c|}{ Sodium Peroxide/Hydroxide Fusion* } \\
\hline $\mathrm{Al}$ & $2.49 \mathrm{E}+00$ & $2.06 \mathrm{E}+00$ & $2.50 \mathrm{E}+00$ & -0.5 \\
\hline $\mathrm{B}$ & $2.57 \mathrm{E}+00$ & $2.37 \mathrm{E}+00$ & $2.69 \mathrm{E}+00$ & -4.5 \\
\hline $\mathrm{Ca}$ & $1.21 \mathrm{E}+00$ & $3.43 \mathrm{E}+00$ & $1.02 \mathrm{E}+00$ & 19.0 \\
\hline $\mathrm{Cr}$ & $6.90 \mathrm{E}-02$ & $3.23 \mathrm{E}+00$ & $6.40 \mathrm{E}-02$ & 7.8 \\
\hline $\mathrm{Cu}$ & $<4.00 \mathrm{E}-02$ & NA & $3.00 \mathrm{E}-03$ & NA \\
\hline $\mathrm{Fe}$ & $9.71 \mathrm{E}+00$ & $2.10 \mathrm{E}+00$ & $9.79 \mathrm{E}+00$ & $\begin{array}{l}-0.8 \\
\end{array}$ \\
\hline K & $2.85 \mathrm{E}+00$ & $6.38 \mathrm{E}+00$ & $2.26 \mathrm{E}+00$ & 26.0 \\
\hline $\mathrm{Li}$ & $1.49 \mathrm{E}+00$ & $2.93 \mathrm{E}+00$ & $1.49 \mathrm{E}+00$ & 0.0 \\
\hline $\mathrm{Mg}$ & 5.34E-01 & $1.87 \mathrm{E}+00$ & $5.20 \mathrm{E}-01$ & 2.8 \\
\hline $\mathrm{Mn}$ & $1.33 \mathrm{E}+00$ & $2.17 \mathrm{E}+00$ & $1.46 \mathrm{E}+00$ & -8.7 \\
\hline $\mathrm{Na}$ & NA & NA & $8.52 \mathrm{E}+00$ & NA \\
\hline $\mathrm{Ni}$ & 7.83E-01 & $3.41 \mathrm{E}+00$ & $8.27 \mathrm{E}-01$ & -5.3 \\
\hline $\mathrm{Si}$ & $2.04 \mathrm{E}+01$ & $2.14 \mathrm{E}+00$ & $2.24 \mathrm{E}+01$ & -8.9 \\
\hline $\mathrm{Ti}$ & 6.62E-01 & $2.48 \mathrm{E}+00$ & $6.90 \mathrm{E}-01$ & -4.1 \\
\hline $\mathrm{U}$ & NA & NA & NA & NA \\
\hline $\mathrm{Zr}$ & NA & NA & $9.60 \mathrm{E}-02$ & NA \\
\hline
\end{tabular}

*All averages are based upon three replicate dissolutions and ICP-AES determinations NA = Not applicable. 
Table 3-6. Continuation of elemental concentrations of ARG standard from ICP-AES analysis of Aqua Regia, DWPF Cold Chem method and Sodium Peroxide/Hydroxide Fusion digestions performed concurrently with SB5 SRAT Receipt. Values are presented on a weight percent $(\mathrm{Wt} \%)$ total solids basis.

\begin{tabular}{|c|c|c|c|c|}
\hline \multicolumn{5}{|c|}{ DWPF Cold Chem Method* } \\
\hline Element & Average & \%RSD & Known Value & $\begin{array}{c}\text { \%Difference } \\
\text { (Measured vs } \\
\text { Known Value) }\end{array}$ \\
\hline $\mathrm{Al}$ & $2.63 \mathrm{E}+00$ & $1.7 \mathrm{E}+00$ & $2.50 \mathrm{E}+00$ & 5.2 \\
\hline $\mathrm{B}$ & $2.80 \mathrm{E}+00$ & $2.3 \mathrm{E}+00$ & $2.69 \mathrm{E}+00$ & 4.2 \\
\hline $\mathrm{Ca}$ & $1.15 \mathrm{E}+00$ & $2.4 \mathrm{E}+00$ & $1.02 \mathrm{E}+00$ & 12.3 \\
\hline $\mathrm{Cr}$ & 6.87E-02 & $4.8 \mathrm{E}+00$ & $6.40 \mathrm{E}-02$ & 7.3 \\
\hline $\mathrm{Cu}$ & $<4.19 \mathrm{E}-02$ & $1.1 \mathrm{E}+00$ & 3.00E-03 & NA \\
\hline $\mathrm{Fe}$ & $1.02 \mathrm{E}+01$ & $2.0 \mathrm{E}+00$ & $9.79 \mathrm{E}+00$ & 4.1 \\
\hline $\mathrm{K}$ & $2.38 \mathrm{E}+00$ & 6.3E-01 & $2.26 \mathrm{E}+00$ & 5.3 \\
\hline $\mathrm{Li}$ & $1.62 \mathrm{E}+00$ & $1.3 \mathrm{E}+00$ & $1.49 \mathrm{E}+00$ & 8.6 \\
\hline Mg & 5.56E-01 & $2.1 \mathrm{E}+00$ & $5.20 \mathrm{E}-01$ & 6.9 \\
\hline Mn & $1.47 \mathrm{E}+00$ & $1.9 \mathrm{E}+00$ & $1.46 \mathrm{E}+00$ & 0.7 \\
\hline $\mathrm{Na}$ & $9.11 \mathrm{E}+00$ & $1.5 \mathrm{E}+00$ & $8.52 \mathrm{E}+00$ & 6.9 \\
\hline $\mathrm{Ni}$ & 8.07E-01 & $1.1 \mathrm{E}+00$ & 8.27E-01 & -2.4 \\
\hline $\mathrm{Si}$ & NA & NA & $2.24 \mathrm{E}+01$ & NA \\
\hline $\mathrm{Ti}$ & 7.26E-01 & $1.7 \mathrm{E}+00$ & $6.90 \mathrm{E}-01$ & 5.2 \\
\hline U & NA & NA & NA & NA \\
\hline $\mathrm{Zr}$ & $1.08 \mathrm{E}-01$ & $1.6 \mathrm{E}+00$ & $9.60 \mathrm{E}-02$ & 12.5 \\
\hline
\end{tabular}

*All averages are based upon three replicate dissolutions and ICP-AES determinations NA = Not applicable. $<\mathrm{MDL}=$ less than minimum detection limit. 
Table 3-7. Elemental concentrations of ARG standard from ICP-AES analysis of Aqua Regia, DWPF Cold Chem method and Sodium Peroxide/Hydroxide Fusion digestions performed concurrently with SB5 SRAT Product. Values are presented on a weight percent $(\mathrm{Wt} \%)$ total solids basis.

\begin{tabular}{|c|c|c|c|c|}
\hline \multicolumn{5}{|l|}{ Aqua Regia* } \\
\hline Element & Average & \%RSD & Standard Value & $\begin{array}{c}\text { \%Difference } \\
\text { (Measured vs } \\
\text { Standard Value) }\end{array}$ \\
\hline $\mathrm{Al}$ & $2.35 \mathrm{E}+00$ & $2.50 \mathrm{E}+00$ & $2.50 \mathrm{E}+00$ & -6.0 \\
\hline $\mathrm{B}$ & $2.63 \mathrm{E}+00$ & $2.69 \mathrm{E}+00$ & $2.69 \mathrm{E}+00$ & -2.4 \\
\hline $\mathrm{Ca}$ & $1.05 \mathrm{E}+00$ & $1.02 \mathrm{E}+00$ & $1.02 \mathrm{E}+00$ & 3.0 \\
\hline $\mathrm{Cr}$ & $6.37 \mathrm{E}-02$ & $6.40 \mathrm{E}-02$ & $6.40 \mathrm{E}-02$ & -0.5 \\
\hline $\mathrm{Cu}$ & $<4.00 \mathrm{E}-02$ & NA & $3.00 \mathrm{E}-03$ & NA \\
\hline $\mathrm{Fe}$ & $9.64 \mathrm{E}+00$ & $9.79 \mathrm{E}+00$ & $9.79 \mathrm{E}+00$ & -1.6 \\
\hline $\mathrm{K}$ & $2.11 \mathrm{E}+00$ & $2.26 \mathrm{E}+00$ & $2.26 \mathrm{E}+00$ & -6.8 \\
\hline $\mathrm{Li}$ & $1.48 \mathrm{E}+00$ & $1.49 \mathrm{E}+00$ & $1.49 \mathrm{E}+00$ & -0.7 \\
\hline $\mathrm{Mg}$ & 5.89E-01 & $5.20 \mathrm{E}-01$ & $5.20 \mathrm{E}-01$ & 13.2 \\
\hline $\mathrm{Mn}$ & $1.41 \mathrm{E}+00$ & $1.46 \mathrm{E}+00$ & $1.46 \mathrm{E}+00$ & -3.4 \\
\hline $\mathrm{Na}$ & $8.14 \mathrm{E}+00$ & $8.52 \mathrm{E}+00$ & $8.52 \mathrm{E}+00$ & -4.5 \\
\hline $\mathrm{Ni}$ & 8.27E-01 & 8.27E-01 & 8.27E-01 & 0.0 \\
\hline $\mathrm{Si}$ & $6.68 \mathrm{E}-01$ & $2.24 \mathrm{E}+01$ & $2.24 \mathrm{E}+01$ & -97.0 \\
\hline $\mathrm{Ti}$ & $5.92 \mathrm{E}-01$ & $6.90 \mathrm{E}-01$ & $6.90 \mathrm{E}-01$ & -14.2 \\
\hline $\mathrm{U}$ & NA & NA & NA & NA \\
\hline $\mathrm{Zr}$ & $6.03 \mathrm{E}-02$ & $9.60 \mathrm{E}-02$ & $9.60 \mathrm{E}-02$ & -37.2 \\
\hline \multicolumn{5}{|c|}{ Sodium Peroxide/Hydroxide Fusion* } \\
\hline $\mathrm{Al}$ & $2.32 \mathrm{E}+00$ & $3.4 \mathrm{E}+00$ & $2.50 \mathrm{E}+00$ & -7.2 \\
\hline B & $2.62 \mathrm{E}+00$ & $3.0 \mathrm{E}+00$ & $2.69 \mathrm{E}+00$ & -2.6 \\
\hline $\mathrm{Ca}$ & $1.06 \mathrm{E}+00$ & $2.5 \mathrm{E}+00$ & $1.02 \mathrm{E}+00$ & 3.9 \\
\hline $\mathrm{Cr}$ & 6.34E-02 & $3.9 \mathrm{E}+00$ & $6.40 \mathrm{E}-02$ & -1.0 \\
\hline $\mathrm{Cu}$ & $<4.00 \mathrm{E}-02$ & NA & 3.00E-03 & NA \\
\hline $\mathrm{Fe}$ & $8.96 \mathrm{E}+00$ & $3.2 \mathrm{E}+00$ & $9.79 \mathrm{E}+00$ & -8.5 \\
\hline $\mathrm{K}$ & $2.17 \mathrm{E}+00$ & $1.6 \mathrm{E}+00$ & $2.26 \mathrm{E}+00$ & -4.0 \\
\hline $\mathrm{Li}$ & $1.48 \mathrm{E}+00$ & $2.4 \mathrm{E}+00$ & $1.49 \mathrm{E}+00$ & -0.7 \\
\hline $\mathrm{Mg}$ & 5.50E-01 & $5.2 \mathrm{E}+00$ & 5.20E-01 & 5.7 \\
\hline $\mathrm{Mn}$ & $1.32 \mathrm{E}+00$ & $3.5 \mathrm{E}+00$ & $1.46 \mathrm{E}+00$ & -9.4 \\
\hline $\mathrm{Ni}$ & 7.90E-01 & $4.2 \mathrm{E}+00$ & 8.27E-01 & -4.4 \\
\hline $\mathrm{Si}$ & $2.04 \mathrm{E}+01$ & $1.3 \mathrm{E}+01$ & $2.24 \mathrm{E}+01$ & -8.8 \\
\hline $\mathrm{Ti}$ & 6.63E-01 & $2.9 \mathrm{E}+00$ & $6.90 \mathrm{E}-01$ & -3.9 \\
\hline $\mathrm{U}$ & NA & NA & NA & NA \\
\hline $\mathrm{Zr}$ & NA & NA & $9.60 \mathrm{E}-02$ & NA \\
\hline
\end{tabular}

*All averages are based upon three replicate dissolutions and ICP-ES determinations NA = Not applicable. 
Table 3-8. Continuation of elemental concentrations of ARG standard from ICP-AES analysis of Aqua Regia, DWPF Cold Chem method and Sodium Peroxide/Hydroxide Fusion digestions performed concurrently with SB5 SRAT Product. Values are presented on a weight percent $(\mathrm{Wt} \%)$ total solids basis.

\begin{tabular}{|c|c|c|c|c|}
\hline \multicolumn{5}{|c|}{ DWPF Cold Chem Method* } \\
\hline Element & Average & \%RSD & Known Value & $\begin{array}{c}\text { \%Difference } \\
\text { (Measured vs } \\
\text { Known Value) }\end{array}$ \\
\hline $\mathrm{Al}$ & $2.58 \mathrm{E}+00$ & $1.7 \mathrm{E}+00$ & $2.50 \mathrm{E}+00$ & 3.2 \\
\hline $\mathrm{B}$ & $2.92 \mathrm{E}+00$ & $4.2 \mathrm{E}+00$ & $2.69 \mathrm{E}+00$ & 8.6 \\
\hline $\mathrm{Ca}$ & $1.03 \mathrm{E}+00$ & $1.2 \mathrm{E}+00$ & $1.02 \mathrm{E}+00$ & 1.3 \\
\hline $\mathrm{Cr}$ & $6.84 \mathrm{E}-02$ & 8.1E-01 & $6.40 \mathrm{E}-02$ & 6.9 \\
\hline $\mathrm{Cu}$ & $<1.10 \mathrm{E}-02$ & NA & $3.00 \mathrm{E}-03$ & NA \\
\hline $\mathrm{Fe}$ & $1.00 \mathrm{E}+01$ & $6.2 \mathrm{E}-01$ & $9.79 \mathrm{E}+00$ & 2.4 \\
\hline $\mathrm{K}$ & $2.24 \mathrm{E}+00$ & $2.1 \mathrm{E}+00$ & $2.26 \mathrm{E}+00$ & -0.8 \\
\hline $\mathrm{Li}$ & $1.51 \mathrm{E}+00$ & $3.2 \mathrm{E}+00$ & $1.49 \mathrm{E}+00$ & 1.2 \\
\hline $\mathrm{Mg}$ & $5.55 \mathrm{E}-01$ & $5.2 \mathrm{E}-01$ & $5.20 \mathrm{E}-01$ & 6.7 \\
\hline Mn & $1.47 \mathrm{E}+00$ & 8.6E-01 & $1.46 \mathrm{E}+00$ & 0.7 \\
\hline $\mathrm{Na}$ & $8.89 \mathrm{E}+00$ & $1.1 \mathrm{E}+00$ & $8.52 \mathrm{E}+00$ & 4.4 \\
\hline $\mathrm{Ni}$ & 8.41E-01 & $2.6 \mathrm{E}-01$ & $8.27 \mathrm{E}-01$ & 1.7 \\
\hline $\mathrm{Si}$ & NA & NA & $2.24 \mathrm{E}+01$ & NA \\
\hline $\mathrm{Ti}$ & 7.13E-01 & $1.6 \mathrm{E}+00$ & 6.90E-01 & 3.4 \\
\hline $\mathrm{U}$ & NA & NA & NA & NA \\
\hline $\mathrm{Zr}$ & $1.05 \mathrm{E}-01$ & $2.0 \mathrm{E}+00$ & $9.60 \mathrm{E}-02$ & 9.9 \\
\hline
\end{tabular}

*All averages are based upon three replicate dissolutions and ICP-AES determinations NA $=$ Not applicable. $<\mathrm{MDL}=$ less than minimum detection limit. 


\subsection{CONCLUSIONS}

The results presented in the memo validate the use of the DWPF CC method for use with the SB5 material. The relative small difference observed between the three digestions (AR, PF and DWPF CC) for elements composing greater than $1 \mathrm{wt} \%$ of the solids (except for Al) indicate the DWPF CC digestion method is sufficient for digesting SB5 process samples. The reason for the discrepancy in the aluminum concentration obtained from the AR digestion compared to the DWPF CC method and PF method is not known. The peroxide fusion digestion method is the best method for digesting aluminosilicates and aluminum hydroxides that otherwise might be insoluble in acid digestions. Given that the measured aluminum concentration in samples digested using the DWPC CC method is statistically equivalent to the measured aluminum concentration in PF digested samples, the DWPF CC method appears to be adequately dissolving aluminum containing species in the SB5 Batch sludge.

However, the SB5 Batch material is to be mixed with the heel of SB4 to form the SB5 Blend that is to be processed at DWPF, and a difficulty was encountered in using the CC method for SB4. ${ }^{6}$ This difficulty brings into question the adequacy of CC for the SB5 Blend (see recommendations below). 
This page intentionally left blank 


\subsection{RECOMMENDATIONS}

The following recommendations are based upon results in this memo:

- A dissolution study should be performed on the WAPS sample by SRNL which consists of the final composition of the sludge (the SB5 Blend).

- Given the heel of SB4 in Tank 40, the DWPF lab should monitor the aluminum concentration in the first 10 SRAT Receipt batches of SB5 using both CC and sodium peroxide/hydroxide fusion to evaluate the adequacy of aluminum recovery by the CC method for this sludge batch.

- SRNL and the DWPF lab should investigate if comparisons between the elemental concentrations of the SME product glass (adjusted for frit addition) obtained by the mixed acid and peroxide fusion digestion and the SRAT Receipt and SRAT Product elemental concentrations obtained via the DWPF CC method provide insight into the adequacy of the CC method for analysis of the SRAT Product. The DWPF lab would need to calcine the SRAT product at $1050^{\circ} \mathrm{C}$ for the best comparison. If a consistent difference in elemental concentrations is revealed, another type of digestion (i.e. sodium peroxide/hydroxide fusion) should be used to determine the concentration of the element in question. Particular emphasis should be placed on monitoring the aluminum concentration in SB5. 
This page intentionally left blank 


\subsection{REFERENCES}

1. C.J. Coleman, “Alkali Fusion Dissolutions of Sludge and Glass for Elemental and Anion Analysis”, ADS Procedure ADS-2502, Rev. 6.

2. C.J. Coleman, “Aqua Regia Dissolution of Sludge for Elemental Analysis”, ADS Procedure ADS-2226, Rev. 7.

3. M.S. Hay, J.M. Pareizs, C.J. Bannochie, M.E. Stone, D.R. Click and D.J. McCabe, "Characterization and Aluminum Dissolution Demonstration With A 3 Liter Tank 51H Sample,” WSRC-STI-2007-00697, Rev. 0; J.A. Pike, "Preliminary Results for Low Temperature Aluminum Removal from Sludge Batch 5,” LWO-LWE-2008-00067 Rev. 0; J.A. Pike and J.M. Gillam, "Flowsheet for Aluminum Removal from Sludge Batch 5," LWO-PIT-2007-00042 Rev. 2.

4. JMP Statistical Discovery Software v 6.0; SAS Institute Inc., Cary, NC, 2002.

5. C.J. Coleman, F.M. Pennebaker, B.H. Burch and D.R. Click, "Evaluation of the DWPF Cold Chem Dissolution Method with DWPF Sludge Batch 3 Simulant”, WSRC-TR-0200496, Rev. 0. See also D.R. Click, "Evaluation of the DWPF Cold Chem Dissolution Method with Tank 7 and Tank 51 Radioactive Sludges”, WSRC-TR-2003-00580. D.R. Click, C.J. Coleman, K.E. Zeigler and T.B. Edwards, "Sludge Batch Four (4) Defense Waste Processing Facility (DWPF) Process Analytical Method Verification”, WSRCSTI-2006-00025 Rev. 0.

6. D.R. Click, C.J. Coleman, K.E. Zeigler and T.B. Edwards, "Sludge Batch Four (4) Defense Waste Processing Facility (DWPF) Process Analytical Method Verification”, WSRC-STI-2006-00025 Rev. 0. 
This page intentionally left blank 


\subsection{ACKNOWLEDGEMENTS}

The authors would like to acknowledge Ramona Galloway and Monica Jenkins. 
This page intentionally left blank 


\subsection{APPENDIX A. SUPPORTING INFORMATION}


Table A1. Measurements Generated by this Study

\begin{tabular}{|c|c|c|c|c|c|c|c|c|c|}
\hline Type & "Digestion & "Sample ID & Element & "Wt\% Measurement & Type & Digestion & "Sample ID & Element & "Wt\% Measurement \\
\hline SRAT Product & CC & 300250343 & $\mathrm{Al}$ & 6.3845 & SRAT Receipt & CC & 300249668 & $\mathrm{Al}$ & 9.5096 \\
\hline SRAT Product & CC & 300250343 & B & 0.155 & SRAT Receipt & CC & 300249668 & B & 0.1347 \\
\hline SRAT Product & $\mathrm{CC}$ & 300250343 & $\mathrm{Ca}$ & 1.0381 & SRAT Receipt & $\mathrm{CC}$ & 300249668 & $\mathrm{Ca}$ & 1.3828 \\
\hline SRAT Product & $\mathrm{CC}$ & 300250343 & $\mathrm{Cr}$ & 0.0388 & SRAT Receipt & CC & 300249668 & $\mathrm{Cr}$ & 0.0421 \\
\hline SRAT Product & $\mathrm{CC}$ & 300250343 & $\mathrm{Cu}$ & 0.0453 & SRAT Receipt & $\mathrm{CC}$ & 300249668 & $\mathrm{Cu}$ & 0.0586 \\
\hline SRAT Product & $\mathrm{CC}$ & 300250343 & $\mathrm{Fe}$ & 12.6715 & SRAT Receipt & CC & 300249668 & $\mathrm{Fe}$ & 15.678 \\
\hline SRAT Product & $\mathrm{CC}$ & 300250343 & $\mathrm{~K}$ & 0.5751 & SRAT Receipt & CC & 300249668 & $\mathrm{~K}$ & 0.6066 \\
\hline SRAT Product & CC & 300250343 & $\mathrm{Li}$ & 0.0279 & SRAT Receipt & CC & 300249668 & $\mathrm{Li}$ & 0.0356 \\
\hline SRAT Product & $\mathrm{CC}$ & 300250343 & $\mathrm{Mg}$ & 0.541 & SRAT Receipt & $\mathrm{CC}$ & 300249668 & $\mathrm{Mg}$ & 0.622 \\
\hline SRAT Product & $\mathrm{CC}$ & 300250343 & $\mathrm{Mn}$ & 3.1581 & SRAT Receipt & CC & 300249668 & $\mathrm{Mn}$ & 3.6702 \\
\hline SRAT Product & CC & 300250343 & $\mathrm{Na}$ & 13.1589 & SRAT Receipt & CC & 300249668 & $\mathrm{Na}$ & 15.678 \\
\hline SRAT Product & $\mathrm{CC}$ & 300250343 & $\mathrm{Ni}$ & 1.9543 & SRAT Receipt & $\mathrm{CC}$ & 300249668 & $\mathrm{Ni}$ & 2.2926 \\
\hline SRAT Product & $\mathrm{CC}$ & 300250343 & $\mathrm{Si}$ & & SRAT Receipt & CC & 300249668 & $\mathrm{Si}$ & - \\
\hline SRAT Product & CC & 300250343 & $\mathrm{Ti}$ & 0.0163 & SRAT Receipt & CC & 300249668 & $\mathrm{Ti}$ & 0.0219 \\
\hline SRAT Product & $\mathrm{CC}$ & 300250343 & $\mathrm{U}$ & 4.8493 & SRAT Receipt & $\mathrm{CC}$ & 300249668 & $\mathrm{U}$ & 5.7058 \\
\hline SRAT Product & CC & 300250343 & $\mathrm{Zr}$ & 0.2179 & SRAT Receipt & $\mathrm{CC}$ & 300249668 & $\mathrm{Zr}$ & 0.2632 \\
\hline SRAT Product & CC & 300250345 & $\mathrm{Al}$ & 6.9276 & SRAT Receipt & CC & 300249670 & $\mathrm{Al}$ & 8.6577 \\
\hline SRAT Product & $\mathrm{CC}$ & 300250345 & $\mathrm{~B}$ & 0.2016 & SRAT Receipt & $\mathrm{CC}$ & 300249670 & $\mathrm{~B}$ & 0.1319 \\
\hline SRAT Product & $\mathrm{CC}$ & 300250345 & $\mathrm{Ca}$ & 1.0941 & SRAT Receipt & CC & 300249670 & $\mathrm{Ca}$ & 1.3238 \\
\hline SRAT Product & CC & 300250345 & $\mathrm{Cr}$ & 0.0414 & SRAT Receipt & CC & 300249670 & $\mathrm{Cr}$ & 0.0424 \\
\hline SRAT Product & CC & 300250345 & $\mathrm{Cu}$ & 0.0466 & SRAT Receipt & CC & 300249670 & $\overline{\mathrm{Cu}}$ & 0.0569 \\
\hline SRAT Product & CC & 300250345 & $\mathrm{Fe}$ & 13.1863 & SRAT Receipt & $\mathrm{CC}$ & 300249670 & $\mathrm{Fe}$ & 14.9496 \\
\hline SRAT Product & $\mathrm{CC}$ & 300250345 & $\mathrm{~K}$ & 0.5638 & SRAT Receipt & $\mathrm{CC}$ & 300249670 & $\mathrm{~K}$ & 0.594 \\
\hline SRAT Product & CC & 300250345 & $\mathrm{Li}$ & 0.0288 & SRAT Receipt & CC & 300249670 & $\mathrm{Li}$ & 0.0295 \\
\hline SRAT Product & CC & 300250345 & $\mathrm{Mg}$ & 0.559 & SRAT Receipt & $\mathrm{CC}$ & 300249670 & $\mathrm{Mg}$ & 0.599 \\
\hline SRAT Product & CC & 300250345 & $\mathrm{Mn}$ & 3.2583 & SRAT Receipt & CC & 300249670 & $\mathrm{Mn}$ & 3.5637 \\
\hline SRAT Product & $\mathrm{CC}$ & 300250345 & $\mathrm{Na}$ & 13.4729 & SRAT Receipt & $\mathrm{CC}$ & 300249670 & $\mathrm{Na}$ & 15.151 \\
\hline SRAT Product & CC & 300250345 & $\mathrm{Ni}$ & 2.0209 & SRAT Receipt & $\mathrm{CC}$ & 300249670 & $\mathrm{Ni}$ & 2.2047 \\
\hline SRAT Product & CC & 300250345 & $\mathrm{Si}$ & & SRAT Receipt & $\mathrm{CC}$ & 300249670 & $\mathrm{Si}$ & - \\
\hline SRAT Product & CC & 300250345 & $\mathrm{Ti}$ & 0.0154 & SRAT Receipt & CC & 300249670 & $\mathrm{Ti}$ & 0.0191 \\
\hline SRAT Product & $\mathrm{CC}$ & 300250345 & $\mathrm{U}$ & 4.8732 & SRAT Receipt & $\mathrm{CC}$ & 300249670 & $\mathrm{U}$ & 5.5872 \\
\hline SRAT Product & $\mathrm{CC}$ & 300250345 & $\mathrm{Zr}$ & 0.2198 & SRAT Receipt & $\mathrm{CC}$ & 300249670 & $\mathrm{Zr}$ & 0.2552 \\
\hline SRAT Product & CC & 300250346 & $\mathrm{Al}$ & 6.5737 & SRAT Receipt & CC & 300249671 & $\mathrm{Al}$ & 8.7637 \\
\hline SRAT Product & CC & 300250346 & $\mathrm{~B}$ & 0.2069 & SRAT Receipt & $\mathrm{CC}$ & 300249671 & $\mathrm{~B}$ & 0.1327 \\
\hline SRAT Product & $\mathrm{CC}$ & 300250346 & $\mathrm{Ca}$ & 1.0601 & SRAT Receipt & CC & 300249671 & $\mathrm{Ca}$ & 1.3373 \\
\hline SRAT Product & CC & 300250346 & $\mathrm{Cr}$ & 0.039 & SRAT Receipt & CC & 300249671 & $\mathrm{Cr}$ & 0.0425 \\
\hline SRAT Product & CC & 300250346 & $\mathrm{Cu}$ & 0.0481 & SRAT Receipt & $\mathrm{CC}$ & 300249671 & $\mathrm{Cu}$ & 0.0572 \\
\hline SRAT Product & $\mathrm{CC}$ & 300250346 & $\mathrm{Fe}$ & 12.9159 & SRAT Receipt & $\mathrm{CC}$ & 300249671 & $\mathrm{Fe}$ & 15.1972 \\
\hline SRAT Product & CC & 300250346 & $\mathrm{~K}$ & 0.5463 & SRAT Receipt & CC & 300249671 & $\mathrm{~K}$ & 0.5978 \\
\hline SRAT Product & $\mathrm{CC}$ & 300250346 & $\mathrm{Li}$ & 0.0298 & SRAT Receipt & $\mathrm{CC}$ & 300249671 & $\mathrm{Li}$ & 0.0317 \\
\hline SRAT Product & CC & 300250346 & $\mathrm{Mg}$ & 0.5463 & SRAT Receipt & CC & 300249671 & $\mathrm{Mg}$ & 0.6079 \\
\hline SRAT Product & CC & 300250346 & $\mathrm{Mn}$ & 3.1943 & SRAT Receipt & CC & 300249671 & $\mathrm{Mn}$ & 3.5865 \\
\hline SRAT Product & CC & 300250346 & $\mathrm{Na}$ & 13.8881 & SRAT Receipt & $\mathrm{CC}$ & 300249671 & $\mathrm{Na}$ & 15.3998 \\
\hline SRAT Product & $\mathrm{CC}$ & 300250346 & $\mathrm{Ni}$ & 1.9999 & SRAT Receipt & $\mathrm{CC}$ & 300249671 & $\mathrm{Ni}$ & 2.2289 \\
\hline SRAT Product & $\mathrm{CC}$ & 300250346 & $\mathrm{Si}$ & & SRAT Receipt & $\mathrm{CC}$ & 300249671 & $\mathrm{Si}$ & . \\
\hline SRAT Product & CC & 300250346 & $\mathrm{Ti}$ & 0.0157 & SRAT Receipt & CC & 300249671 & $\mathrm{Ti}$ & 0.0189 \\
\hline SRAT Product & CC & 300250346 & $\mathrm{U}$ & 4.8608 & SRAT Receipt & $\mathrm{CC}$ & 300249671 & $\mathrm{U}$ & 5.6229 \\
\hline SRAT Product & CC & 300250346 & $\mathrm{Zr}$ & 0.2171 & SRAT Receipt & CC & 300249671 & $\mathrm{Zr}$ & 0.2589 \\
\hline SRAT Product & CC & 300250348 & $\mathrm{Al}$ & 6.9311 & SRAT Receipt & CC & 300249673 & $\mathrm{Al}$ & 8.5403 \\
\hline SRAT Product & $\mathrm{CC}$ & 300250348 & $\mathrm{~B}$ & 0.2314 & SRAT Receipt & $\mathrm{CC}$ & 300249673 & $\mathrm{~B}$ & 0.134 \\
\hline SRAT Product & CC & 300250348 & $\mathrm{Ca}$ & 1.0836 & SRAT Receipt & $\mathrm{CC}$ & 300249673 & $\mathrm{Ca}$ & 1.3399 \\
\hline SRAT Product & CC & 300250348 & $\mathrm{Cr}$ & 0.0397 & SRAT Receipt & CC & 300249673 & $\mathrm{Cr}$ & 0.0427 \\
\hline SRAT Product & $\mathrm{CC}$ & 300250348 & $\mathrm{Cu}$ & 0.0503 & SRAT Receipt & $\mathrm{CC}$ & 300249673 & $\mathrm{Cu}$ & 0.0573 \\
\hline SRAT Product & $\mathrm{CC}$ & 300250348 & $\mathrm{Fe}$ & 13.2278 & SRAT Receipt & $\mathrm{CC}$ & 300249673 & $\mathrm{Fe}$ & 15.2396 \\
\hline SRAT Product & CC & 300250348 & $\mathrm{~K}$ & 0.576 & SRAT Receipt & CC & 300249673 & $\mathrm{~K}$ & 0.6034 \\
\hline SRAT Product & CC & 300250348 & $\mathrm{Li}$ & 0.029 & SRAT Receipt & CC & 300249673 & $\mathrm{Li}$ & 0.0278 \\
\hline SRAT Product & CC & 300250348 & $\mathrm{Mg}$ & 0.5564 & SRAT Receipt & CC & 300249673 & $\mathrm{Mg}$ & 0.6086 \\
\hline SRAT Product & CC & 300250348 & $\mathrm{Mn}$ & 3.2459 & SRAT Receipt & CC & 300249673 & $\mathrm{Mn}$ & 3.6207 \\
\hline SRAT Product & $\mathrm{CC}$ & 300250348 & $\mathrm{Na}$ & 13.7647 & SRAT Receipt & CC & 300249673 & $\mathrm{Na}$ & 15.4953 \\
\hline SRAT Product & CC & 300250348 & $\mathrm{Ni}$ & 2.0452 & SRAT Receipt & CC & 300249673 & $\mathrm{Ni}$ & 2.2501 \\
\hline SRAT Product & CC & 300250348 & $\mathrm{Si}$ & & SRAT Receipt & CC & 300249673 & $\mathrm{Si}$ & \\
\hline SRAT Product & CC & 300250348 & $\mathrm{Ti}$ & 0.0158 & SRAT Receipt & $\mathrm{CC}$ & 300249673 & $\mathrm{Ti}$ & 0.0194 \\
\hline
\end{tabular}


Table A1. Measurements Generated by this Study

\begin{tabular}{|c|c|c|c|c|c|c|c|c|c|}
\hline Type & Digestion & Sample ID & Element & Wt\% Measurement & Type & Digestion & Sample ID & Element & Wt\% Measurement \\
\hline SRAT Product & $\mathrm{CC}$ & 300250348 & $\mathrm{U}$ & 4.8811 & SRAT Receipt & $\mathrm{CC}$ & 300249673 & $\mathrm{U}$ & 5.7788 \\
\hline SRAT Product & $\mathrm{CC}$ & 300250348 & $\mathrm{Zr}$ & 0.2226 & SRAT Receipt & $\mathrm{CC}$ & 300249673 & $\mathrm{Zr}$ & 0.2598 \\
\hline SRAT Product & $\mathrm{PF}$ & 300250136 & $\mathrm{Al}$ & 7.07 & SRAT Receipt & PF & 300249476 & $\mathrm{Al}$ & 8.98 \\
\hline SRAT Product & $\mathrm{PF}$ & 300250136 & $\mathrm{~B}$ & 0.0227 & SRAT Receipt & $\mathrm{PF}$ & 300249476 & $\mathrm{~B}$ & 0.0261 \\
\hline SRAT Product & $\mathrm{PF}$ & 300250136 & $\mathrm{Ca}$ & 1.07 & SRAT Receipt & $\mathrm{PF}$ & 300249476 & $\mathrm{Ca}$ & 1.35 \\
\hline SRAT Product & $\mathrm{PF}$ & 300250136 & $\mathrm{Cr}$ & 0.0372 & SRAT Receipt & PF & 300249476 & $\mathrm{Cr}$ & 0.365 \\
\hline SRAT Product & $\mathrm{PF}$ & 300250136 & $\mathrm{Cu}$ & 0.0449 & SRAT Receipt & $\mathrm{PF}$ & 300249476 & $\mathrm{Cu}$ & 0.0624 \\
\hline SRAT Product & $\mathrm{PF}$ & 300250136 & $\mathrm{Fe}$ & 12.2 & SRAT Receipt & $\mathrm{PF}$ & 300249476 & $\mathrm{Fe}$ & 17.5 \\
\hline SRAT Product & $\mathrm{PF}$ & 300250136 & $\mathrm{~K}$ & 0.51 & SRAT Receipt & PF & 300249476 & $\mathrm{~K}$ & 0.846 \\
\hline SRAT Product & $\mathrm{PF}$ & 300250136 & $\mathrm{Li}$ & 0.0305 & SRAT Receipt & PF & 300249476 & $\mathrm{Li}$ & 0.035 \\
\hline SRAT Product & $\mathrm{PF}$ & 300250136 & $\mathrm{Mg}$ & 0.512 & SRAT Receipt & PF & 300249476 & $\mathrm{Mg}$ & 0.598 \\
\hline SRAT Product & $\mathrm{PF}$ & 300250136 & $\mathrm{Mn}$ & 2.98 & SRAT Receipt & PF & 300249476 & $\mathrm{Mn}$ & 3.59 \\
\hline SRAT Product & $\mathrm{PF}$ & 300250136 & $\mathrm{Na}$ & . & SRAT Receipt & $\mathrm{PF}$ & 300249476 & $\mathrm{Na}$ & . \\
\hline SRAT Product & $\mathrm{PF}$ & 300250136 & $\mathrm{Ni}$ & 1.92 & SRAT Receipt & $\mathrm{PF}$ & 300249476 & $\mathrm{Ni}$ & 2.43 \\
\hline SRAT Product & $\mathrm{PF}$ & 300250136 & $\mathrm{Si}$ & 0.765 & SRAT Receipt & $\mathrm{PF}$ & 300249476 & $\mathrm{Si}$ & 0.922 \\
\hline SRAT Product & $\mathrm{PF}$ & 300250136 & $\mathrm{Ti}$ & 0.0172 & SRAT Receipt & $\mathrm{PF}$ & 300249476 & $\mathrm{Ti}$ & 0.0203 \\
\hline SRAT Product & $\mathrm{PF}$ & 300250136 & $\mathrm{U}$ & 4.87 & SRAT Receipt & $\mathrm{PF}$ & 300249476 & $\mathrm{U}$ & 5.56 \\
\hline SRAT Product & $\mathrm{PF}$ & 300250136 & $\mathrm{Zr}$ &. & SRAT Receipt & $\mathrm{PF}$ & 300249476 & $\mathrm{Zr}$ &. \\
\hline SRAT Product & $\mathrm{PF}$ & 300250138 & $\mathrm{Al}$ & 7.22 & SRAT Receipt & PF & 300249478 & $\mathrm{Al}$ & 9.12 \\
\hline SRAT Product & $\mathrm{PF}$ & 300250138 & $\mathrm{~B}$ & 0.024 & SRAT Receipt & PF & 300249478 & $\mathrm{~B}$ & 0.0263 \\
\hline SRAT Product & $\mathrm{PF}$ & 300250138 & $\mathrm{Ca}$ & 1.09 & SRAT Receipt & PF & 300249478 & $\mathrm{Ca}$ & 1.28 \\
\hline SRAT Product & $\mathrm{PF}$ & 300250138 & $\mathrm{Cr}$ & 0.038 & SRAT Receipt & PF & 300249478 & $\mathrm{Cr}$ & 0.105 \\
\hline SRAT Product & $\mathrm{PF}$ & 300250138 & $\mathrm{Cu}$ & 0.0472 & SRAT Receipt & $\mathrm{PF}$ & 300249478 & $\mathrm{Cu}$ & 0.0607 \\
\hline SRAT Product & $\mathrm{PF}$ & 300250138 & $\mathrm{Fe}$ & 12.5 & SRAT Receipt & $\mathrm{PF}$ & 300249478 & $\mathrm{Fe}$ & 16.6 \\
\hline SRAT Product & $\mathrm{PF}$ & 300250138 & $\mathrm{~K}$ & 0.539 & SRAT Receipt & PF & 300249478 & $\mathrm{~K}$ & 0.802 \\
\hline SRAT Product & $\mathrm{PF}$ & 300250138 & $\mathrm{Li}$ & 0.0352 & SRAT Receipt & $\mathrm{PF}$ & 300249478 & $\mathrm{Li}$ & 0.0393 \\
\hline SRAT Product & $\mathrm{PF}$ & 300250138 & $\mathrm{Mg}$ & 0.542 & SRAT Receipt & $\mathrm{PF}$ & 300249478 & $\mathrm{Mg}$ & 0.605 \\
\hline SRAT Product & $\mathrm{PF}$ & 300250138 & $\mathrm{Mn}$ & 3.05 & SRAT Receipt & PF & 300249478 & $\mathrm{Mn}$ & 3.63 \\
\hline SRAT Product & $\mathrm{PF}$ & 300250138 & $\mathrm{Na}$ & . & SRAT Receipt & $\mathrm{PF}$ & 300249478 & $\mathrm{Na}$ & . \\
\hline SRAT Product & $\mathrm{PF}$ & 300250138 & $\mathrm{Ni}$ & 1.96 & SRAT Receipt & $\mathrm{PF}$ & 300249478 & $\mathrm{Ni}$ & 2.39 \\
\hline SRAT Product & $\mathrm{PF}$ & 300250138 & $\mathrm{Si}$ & 0.811 & SRAT Receipt & $\mathrm{PF}$ & 300249478 & $\mathrm{Si}$ & 0.943 \\
\hline SRAT Product & $\mathrm{PF}$ & 300250138 & $\mathrm{Ti}$ & 0.0173 & SRAT Receipt & $\mathrm{PF}$ & 300249478 & $\mathrm{Ti}$ & 0.0207 \\
\hline SRAT Product & $\mathrm{PF}$ & 300250138 & $\mathrm{U}$ & 4.98 & SRAT Receipt & PF & 300249478 & $\mathrm{U}$ & 5.52 \\
\hline SRAT Product & $\mathrm{PF}$ & 300250138 & $\mathrm{Zr}$ & . & SRAT Receipt & PF & 300249478 & $\mathrm{Zr}$ & \\
\hline SRAT Product & $\mathrm{PF}$ & 300250139 & $\mathrm{Al}$ & 6.88 & SRAT Receipt & $\mathrm{PF}$ & 300249479 & $\mathrm{Al}$ & 8.8 \\
\hline SRAT Product & $\mathrm{PF}$ & 300250139 & $\mathrm{~B}$ & 0.0227 & SRAT Receipt & $\mathrm{PF}$ & 300249479 & $\mathrm{~B}$ & 0.0264 \\
\hline SRAT Product & $\mathrm{PF}$ & 300250139 & $\mathrm{Ca}$ & 1.02 & SRAT Receipt & $\mathrm{PF}$ & 300249479 & $\mathrm{Ca}$ & 1.36 \\
\hline SRAT Product & $\mathrm{PF}$ & 300250139 & $\mathrm{Cr}$ & 0.0431 & SRAT Receipt & $\mathrm{PF}$ & 300249479 & $\mathrm{Cr}$ & 0.049 \\
\hline SRAT Product & $\mathrm{PF}$ & 300250139 & $\mathrm{Cu}$ & 0.0445 & SRAT Receipt & $\mathrm{PF}$ & 300249479 & $\mathrm{Cu}$ & 0.0584 \\
\hline SRAT Product & $\mathrm{PF}$ & 300250139 & $\mathrm{Fe}$ & 12 & SRAT Receipt & $\mathrm{PF}$ & 300249479 & $\mathrm{Fe}$ & 16 \\
\hline SRAT Product & $\mathrm{PF}$ & 300250139 & $\mathrm{~K}$ & 0.512 & SRAT Receipt & $\mathrm{PF}$ & 300249479 & $\mathrm{~K}$ & 0.779 \\
\hline SRAT Product & $\mathrm{PF}$ & 300250139 & $\mathrm{Li}$ & 0.0301 & SRAT Receipt & PF & 300249479 & $\mathrm{Li}$ & 0.034 \\
\hline SRAT Product & $\mathrm{PF}$ & 300250139 & $\mathrm{Mg}$ & 0.509 & SRAT Receipt & $\mathrm{PF}$ & 300249479 & $\mathrm{Mg}$ & 0.607 \\
\hline SRAT Product & $\mathrm{PF}$ & 300250139 & $\mathrm{Mn}$ & 2.9 & SRAT Receipt & $\mathrm{PF}$ & 300249479 & $\mathrm{Mn}$ & 3.57 \\
\hline SRAT Product & $\mathrm{PF}$ & 300250139 & $\mathrm{Na}$ & . & SRAT Receipt & $\mathrm{PF}$ & 300249479 & $\mathrm{Na}$ & . \\
\hline SRAT Product & $\mathrm{PF}$ & 300250139 & $\mathrm{Ni}$ & 1.87 & SRAT Receipt & $\mathrm{PF}$ & 300249479 & $\mathrm{Ni}$ & 2.34 \\
\hline SRAT Product & $\mathrm{PF}$ & 300250139 & $\mathrm{Si}$ & 0.714 & SRAT Receipt & $\mathrm{PF}$ & 300249479 & $\mathrm{Si}$ & 0.908 \\
\hline SRAT Product & $\mathrm{PF}$ & 300250139 & $\mathrm{Ti}$ & 0.0161 & SRAT Receipt & PF & 300249479 & $\mathrm{Ti}$ & 0.02 \\
\hline SRAT Product & $\mathrm{PF}$ & 300250139 & $\mathrm{U}$ & 4.8 & SRAT Receipt & $\mathrm{PF}$ & 300249479 & $\mathrm{U}$ & 5.57 \\
\hline SRAT Product & $\mathrm{PF}$ & 300250139 & $\mathrm{Zr}$ & . & SRAT Receipt & PF & 300249479 & $\mathrm{Zr}$ & . \\
\hline SRAT Product & $\mathrm{PF}$ & 300250141 & $\mathrm{Al}$ & 7.37 & SRAT Receipt & $\mathrm{PF}$ & 300249481 & $\mathrm{Al}$ & 8.75 \\
\hline SRAT Product & $\mathrm{PF}$ & 300250141 & $\mathrm{~B}$ & 0.0243 & SRAT Receipt & $\mathrm{PF}$ & 300249481 & $\mathrm{~B}$ & 0.026 \\
\hline SRAT Product & $\mathrm{PF}$ & 300250141 & $\mathrm{Ca}$ & 1.11 & SRAT Receipt & $\mathrm{PF}$ & 300249481 & $\mathrm{Ca}$ & 1.36 \\
\hline SRAT Product & $\mathrm{PF}$ & 300250141 & $\mathrm{Cr}$ & 0.0414 & SRAT Receipt & $\mathrm{PF}$ & 300249481 & $\mathrm{Cr}$ & 0.0461 \\
\hline SRAT Product & $\mathrm{PF}$ & 300250141 & $\mathrm{Cu}$ & 0.049 & SRAT Receipt & $\mathrm{PF}$ & 300249481 & $\mathrm{Cu}$ & 0.0594 \\
\hline SRAT Product & $\mathrm{PF}$ & 300250141 & $\mathrm{Fe}$ & 12.9 & SRAT Receipt & $\mathrm{PF}$ & 300249481 & $\mathrm{Fe}$ & 15.8 \\
\hline SRAT Product & $\mathrm{PF}$ & 300250141 & $\mathrm{~K}$ & 0.548 & SRAT Receipt & $\mathrm{PF}$ & 300249481 & $\mathrm{~K}$ & 0.712 \\
\hline SRAT Product & $\mathrm{PF}$ & 300250141 & $\mathrm{Li}$ & 0.0315 & SRAT Receipt & PF & 300249481 & $\mathrm{Li}$ & 0.0366 \\
\hline SRAT Product & $\mathrm{PF}$ & 300250141 & $\mathrm{Mg}$ & 0.538 & SRAT Receipt & PF & 300249481 & $\mathrm{Mg}$ & 0.604 \\
\hline SRAT Product & $\mathrm{PF}$ & 300250141 & $\mathrm{Mn}$ & 3.14 & SRAT Receipt & $\mathrm{PF}$ & 300249481 & $\mathrm{Mn}$ & 3.53 \\
\hline SRAT Product & $\mathrm{PF}$ & 300250141 & $\mathrm{Na}$ & . & SRAT Receipt & $\mathrm{PF}$ & 300249481 & $\mathrm{Na}$ & . \\
\hline SRAT Product & $\mathrm{PF}$ & 300250141 & $\mathrm{Ni}$ & 2.01 & SRAT Receipt & $\mathrm{PF}$ & 300249481 & $\mathrm{Ni}$ & 2.3 \\
\hline SRAT Product & $\mathrm{PF}$ & 300250141 & $\mathrm{Si}$ & 0.738 & SRAT Receipt & $\mathrm{PF}$ & 300249481 & $\mathrm{Si}$ & 0.917 \\
\hline
\end{tabular}


Table A1. Measurements Generated by this Study

\begin{tabular}{|c|c|c|c|c|c|c|c|c|c|}
\hline Type & Digestion & Sample ID & Element & Wt\% Measurement & Type & Digestion & Sample ID & Element & Wt\% Measurement \\
\hline SRAT Product & $\mathrm{PF}$ & 300250141 & $\mathrm{Ti}$ & 0.0177 & SRAT Receipt & $\mathrm{PF}$ & 300249481 & $\mathrm{Ti}$ & 0.0202 \\
\hline SRAT Product & $\mathrm{PF}$ & 300250141 & $\mathrm{U}$ & 5.12 & SRAT Receipt & $\mathrm{PF}$ & 300249481 & $\mathrm{U}$ & 5.63 \\
\hline SRAT Product & $\mathrm{PF}$ & 300250141 & $\mathrm{Zr}$ & & SRAT Receipt & PF & 300249481 & $\mathrm{Zr}$ & \\
\hline SRAT Product & AR & 300250117 & $\mathrm{Al}$ & 5.46 & SRAT Receipt & $\mathrm{AR}$ & 300249460 & $\mathrm{Al}$ & 6.98 \\
\hline SRAT Product & AR & 300250117 & $\mathrm{~B}$ & 0.0244 & SRAT Receipt & $\mathrm{AR}$ & 300249460 & $\mathrm{~B}$ & 0.0261 \\
\hline SRAT Product & $\mathrm{AR}$ & 300250117 & $\mathrm{Ca}$ & 1.15 & SRAT Receipt & $\mathrm{AR}$ & 300249460 & $\mathrm{Ca}$ & 1.31 \\
\hline SRAT Product & $\mathrm{AR}$ & 300250117 & $\mathrm{Cr}$ & 0.0398 & SRAT Receipt & $\mathrm{AR}$ & 300249460 & $\mathrm{Cr}$ & 0.0461 \\
\hline SRAT Product & $\mathrm{AR}$ & 300250117 & $\mathrm{Cu}$ & 0.0536 & SRAT Receipt & $\mathrm{AR}$ & 300249460 & $\mathrm{Cu}$ & 0.0658 \\
\hline SRAT Product & AR & 300250117 & $\mathrm{Fe}$ & 13.7 & SRAT Receipt & $\mathrm{AR}$ & 300249460 & $\mathrm{Fe}$ & 16 \\
\hline SRAT Product & AR & 300250117 & $\mathrm{~K}$ & 0.11 & SRAT Receipt & AR & 300249460 & $\mathrm{~K}$ & 0.117 \\
\hline SRAT Product & $\mathrm{AR}$ & 300250117 & $\mathrm{Li}$ & 0.0293 & SRAT Receipt & $\mathrm{AR}$ & 300249460 & $\mathrm{Li}$ & 0.0331 \\
\hline SRAT Product & $\mathrm{AR}$ & 300250117 & $\mathrm{Mg}$ & 0.616 & SRAT Receipt & $\mathrm{AR}$ & 300249460 & $\mathrm{Mg}$ & 0.676 \\
\hline SRAT Product & AR & 300250117 & $\mathrm{Mn}$ & 3.2 & SRAT Receipt & $\mathrm{AR}$ & 300249460 & $\mathrm{Mn}$ & 3.74 \\
\hline SRAT Product & AR & 300250117 & $\mathrm{Na}$ & 13 & SRAT Receipt & AR & 300249460 & $\mathrm{Na}$ & 15.2 \\
\hline SRAT Product & $\mathrm{AR}$ & 300250117 & $\mathrm{Ni}$ & 2.08 & SRAT Receipt & $\mathrm{AR}$ & 300249460 & $\mathrm{Ni}$ & 2.32 \\
\hline SRAT Product & AR & 300250117 & $\mathrm{Si}$ & 0.367 & SRAT Receipt & $\mathrm{AR}$ & 300249460 & $\mathrm{Si}$ & 0.481 \\
\hline SRAT Product & $\mathrm{AR}$ & 300250117 & $\mathrm{Ti}$ & 0.0138 & SRAT Receipt & $\mathrm{AR}$ & 300249460 & $\mathrm{Ti}$ & 0.0197 \\
\hline SRAT Product & $\mathrm{AR}$ & 300250117 & $\mathrm{U}$ & 4.89 & SRAT Receipt & $\mathrm{AR}$ & 300249460 & $\mathrm{U}$ & 5.59 \\
\hline SRAT Product & $\mathrm{AR}$ & 300250117 & $\mathrm{Zr}$ & 0.0934 & SRAT Receipt & $\mathrm{AR}$ & 300249460 & $\mathrm{Zr}$ & 0.144 \\
\hline SRAT Product & $\mathrm{AR}$ & 300250119 & $\mathrm{Al}$ & 5.15 & SRAT Receipt & $\mathrm{AR}$ & 300249462 & $\mathrm{Al}$ & 7.53 \\
\hline SRAT Product & $\mathrm{AR}$ & 300250119 & $\mathrm{~B}$ & 0.0241 & SRAT Receipt & $\mathrm{AR}$ & 300249462 & $\mathrm{~B}$ & 0.0269 \\
\hline SRAT Product & AR & 300250119 & $\mathrm{Ca}$ & 1.16 & SRAT Receipt & $\mathrm{AR}$ & 300249462 & $\mathrm{Ca}$ & 1.34 \\
\hline SRAT Product & AR & 300250119 & $\mathrm{Cr}$ & 0.0393 & SRAT Receipt & AR & 300249462 & $\mathrm{Cr}$ & 0.0488 \\
\hline SRAT Product & $\mathrm{AR}$ & 300250119 & $\mathrm{Cu}$ & 0.0554 & SRAT Receipt & $\mathrm{AR}$ & 300249462 & $\mathrm{Cu}$ & 0.0668 \\
\hline SRAT Product & AR & 300250119 & $\mathrm{Fe}$ & 13.8 & SRAT Receipt & $\mathrm{AR}$ & 300249462 & $\mathrm{Fe}$ & 16.5 \\
\hline SRAT Product & AR & 300250119 & $\mathrm{~K}$ & 0.108 & SRAT Receipt & $\mathrm{AR}$ & 300249462 & $\mathrm{~K}$ & 0.121 \\
\hline SRAT Product & $\mathrm{AR}$ & 300250119 & $\mathrm{Li}$ & 0.029 & SRAT Receipt & $\mathrm{AR}$ & 300249462 & $\mathrm{Li}$ & 0.0371 \\
\hline SRAT Product & $\mathrm{AR}$ & 300250119 & $\mathrm{Mg}$ & 0.616 & SRAT Receipt & $\mathrm{AR}$ & 300249462 & $\mathrm{Mg}$ & 0.697 \\
\hline SRAT Product & $\mathrm{AR}$ & 300250119 & $\mathrm{Mn}$ & 3.21 & SRAT Receipt & $\mathrm{AR}$ & 300249462 & $\mathrm{Mn}$ & 3.83 \\
\hline SRAT Product & AR & 300250119 & $\mathrm{Na}$ & 13 & SRAT Receipt & AR & 300249462 & $\mathrm{Na}$ & 15.6 \\
\hline SRAT Product & $\mathrm{AR}$ & 300250119 & $\mathrm{Ni}$ & 2.1 & SRAT Receipt & $\mathrm{AR}$ & 300249462 & $\mathrm{Ni}$ & 2.35 \\
\hline SRAT Product & $\mathrm{AR}$ & 300250119 & $\mathrm{Si}$ & 0.401 & SRAT Receipt & $\mathrm{AR}$ & 300249462 & $\mathrm{Si}$ & 0.514 \\
\hline SRAT Product & AR & 300250119 & $\mathrm{Ti}$ & 0.0144 & SRAT Receipt & $\mathrm{AR}$ & 300249462 & $\mathrm{Ti}$ & 0.0197 \\
\hline SRAT Product & $\mathrm{AR}$ & 300250119 & $\mathrm{U}$ & 4.97 & SRAT Receipt & $\mathrm{AR}$ & 300249462 & $\mathrm{U}$ & 5.7 \\
\hline SRAT Product & AR & 300250119 & $\mathrm{Zr}$ & 0.111 & SRAT Receipt & $\mathrm{AR}$ & 300249462 & $\mathrm{Zr}$ & 0.0925 \\
\hline SRAT Product & $\mathrm{AR}$ & 300250120 & $\mathrm{Al}$ & 5.59 & SRAT Receipt & $\mathrm{AR}$ & 300249463 & $\mathrm{Al}$ & 8.09 \\
\hline SRAT Product & AR & 300250120 & $\mathrm{~B}$ & 0.0241 & SRAT Receipt & $\mathrm{AR}$ & 300249463 & $\mathrm{~B}$ & 0.0258 \\
\hline SRAT Product & $\mathrm{AR}$ & 300250120 & $\mathrm{Ca}$ & 1.08 & SRAT Receipt & $\mathrm{AR}$ & 300249463 & $\mathrm{Ca}$ & 1.28 \\
\hline SRAT Product & AR & 300250120 & $\mathrm{Cr}$ & 0.0379 & SRAT Receipt & $\mathrm{AR}$ & 300249463 & $\mathrm{Cr}$ & 0.0457 \\
\hline SRAT Product & $\mathrm{AR}$ & 300250120 & $\mathrm{Cu}$ & 0.0502 & SRAT Receipt & $\mathrm{AR}$ & 300249463 & $\mathrm{Cu}$ & 0.0634 \\
\hline SRAT Product & AR & 300250120 & $\mathrm{Fe}$ & 12.8 & SRAT Receipt & $\mathrm{AR}$ & 300249463 & $\mathrm{Fe}$ & 15.7 \\
\hline SRAT Product & AR & 300250120 & $\mathrm{~K}$ & 0.109 & SRAT Receipt & $\mathrm{AR}$ & 300249463 & $\mathrm{~K}$ & 0.116 \\
\hline SRAT Product & $\mathrm{AR}$ & 300250120 & $\mathrm{Li}$ & 0.0272 & SRAT Receipt & $\mathrm{AR}$ & 300249463 & $\mathrm{Li}$ & 0.0369 \\
\hline SRAT Product & AR & 300250120 & $\mathrm{Mg}$ & 0.578 & SRAT Receipt & $\mathrm{AR}$ & 300249463 & $\mathrm{Mg}$ & 0.667 \\
\hline SRAT Product & $\mathrm{AR}$ & 300250120 & Mn & 2.98 & SRAT Receipt & $\mathrm{AR}$ & 300249463 & $\mathrm{Mn}$ & 3.67 \\
\hline SRAT Product & AR & 300250120 & $\mathrm{Na}$ & 12.1 & SRAT Receipt & $\mathrm{AR}$ & 300249463 & $\mathrm{Na}$ & 15 \\
\hline SRAT Product & AR & 300250120 & $\mathrm{Ni}$ & 1.95 & SRAT Receipt & $\mathrm{AR}$ & 300249463 & $\mathrm{Ni}$ & 2.28 \\
\hline SRAT Product & $\mathrm{AR}$ & 300250120 & $\mathrm{Si}$ & 0.247 & SRAT Receipt & $\mathrm{AR}$ & 300249463 & $\mathrm{Si}$ & 0.217 \\
\hline SRAT Product & AR & 300250120 & $\mathrm{Ti}$ & 0.0135 & SRAT Receipt & $\mathrm{AR}$ & 300249463 & $\mathrm{Ti}$ & 0.0202 \\
\hline SRAT Product & AR & 300250120 & $\mathrm{U}$ & 4.55 & SRAT Receipt & $\mathrm{AR}$ & 300249463 & $\mathrm{U}$ & 5.49 \\
\hline SRAT Product & $\mathrm{AR}$ & 300250120 & $\mathrm{Zr}$ & 0.1 & SRAT Receipt & $\mathrm{AR}$ & 300249463 & $\mathrm{Zr}$ & 0.117 \\
\hline SRAT Product & AR & 300250122 & $\mathrm{Al}$ & 5.29 & SRAT Receipt & $\mathrm{AR}$ & 300249465 & $\mathrm{Al}$ & 7.88 \\
\hline SRAT Product & AR & 300250122 & $\mathrm{~B}$ & 0.0236 & SRAT Receipt & $\mathrm{AR}$ & 300249465 & $\mathrm{~B}$ & 0.0262 \\
\hline SRAT Product & AR & 300250122 & $\mathrm{Ca}$ & 1.14 & SRAT Receipt & $\mathrm{AR}$ & 300249465 & $\mathrm{Ca}$ & 1.3 \\
\hline SRAT Product & $\mathrm{AR}$ & 300250122 & $\mathrm{Cr}$ & 0.041 & SRAT Receipt & $\mathrm{AR}$ & 300249465 & $\mathrm{Cr}$ & 0.046 \\
\hline SRAT Product & AR & 300250122 & $\mathrm{Cu}$ & 0.0502 & SRAT Receipt & $\mathrm{AR}$ & 300249465 & $\mathrm{Cu}$ & 0.0621 \\
\hline SRAT Product & AR & 300250122 & $\mathrm{Fe}$ & 13.7 & SRAT Receipt & $\mathrm{AR}$ & 300249465 & $\mathrm{Fe}$ & 15.9 \\
\hline SRAT Product & $\mathrm{AR}$ & 300250122 & $\mathrm{~K}$ & 0.106 & SRAT Receipt & $\mathrm{AR}$ & 300249465 & $\mathrm{~K}$ & 0.118 \\
\hline SRAT Product & $\mathrm{AR}$ & 300250122 & $\mathrm{Li}$ & 0.029 & SRAT Receipt & $\mathrm{AR}$ & 300249465 & $\mathrm{Li}$ & 0.0343 \\
\hline SRAT Product & $\mathrm{AR}$ & 300250122 & $\mathrm{Mg}$ & 0.607 & SRAT Receipt & $\mathrm{AR}$ & 300249465 & $\mathrm{Mg}$ & 0.683 \\
\hline SRAT Product & $\mathrm{AR}$ & 300250122 & $\mathrm{Mn}$ & 3.19 & SRAT Receipt & $\mathrm{AR}$ & 300249465 & $\mathrm{Mn}$ & 3.7 \\
\hline SRAT Product & $\mathrm{AR}$ & 300250122 & $\mathrm{Na}$ & 12.9 & SRAT Receipt & $\mathrm{AR}$ & 300249465 & $\mathrm{Na}$ & 15.1 \\
\hline SRAT Product & AR & 300250122 & $\mathrm{Ni}$ & 2.08 & SRAT Receipt & $\mathrm{AR}$ & 300249465 & $\mathrm{Ni}$ & 2.32 \\
\hline
\end{tabular}


Table A1. Measurements Generated by this Study

\begin{tabular}{|c|c|c|c|c|c|c|c|c|c|}
\hline Type & Digestion & S Sample ID & Element & Wt\% Measurement & Type & Digestion & "Sample ID & Element & Wt\% Measurement \\
\hline SRAT Product & $\mathrm{AR}$ & 300250122 & $\mathrm{Si}$ & 0.359 & SRAT Receipt & $\mathrm{AR}$ & 300249465 & $\mathrm{Si}$ & 0.375 \\
\hline SRAT Product & $\mathrm{AR}$ & 300250122 & $\mathrm{Ti}$ & 0.0134 & SRAT Receipt & $\mathrm{AR}$ & 300249465 & $\mathrm{Ti}$ & 0.0189 \\
\hline SRAT Product & AR & 300250122 & $\overline{\mathrm{U}}$ & 4.88 & SRAT Receipt & AR & 300249465 & $\mathrm{U}$ & 5.52 \\
\hline SRAT Product & $\mathrm{AR}$ & 300250122 & $\mathrm{Zr}$ & 0.0894 & SRAT Receipt & $\mathrm{AR}$ & 300249465 & $\mathrm{Zr}$ & 0.0129 \\
\hline
\end{tabular}




\section{Exhibit A1. Statistical Comparisons of Digestion Methods}

Wt\% Measurement By Digestion Type=SRAT Product, Element=Al

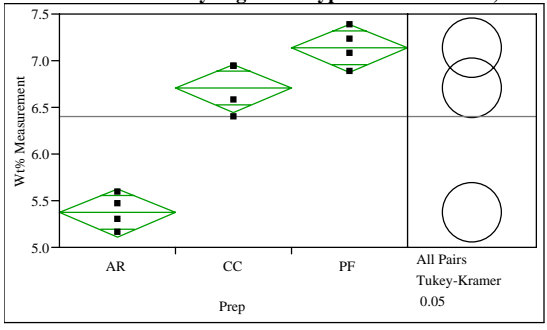

Means Comparisons

$\begin{array}{ll}\text { Level } & \text { Mean } \\ \text { PF } & \text { A } 7.1350000\end{array}$

$\begin{array}{lll}\text { CC } & \text { A } & 6.7042332 \\ \text { AR } & \text { B } 5.372500\end{array}$

Levels not connected by same letter are significantly different.

Wt\% Measurement By Digestion Type=SRAT Product, Element=B

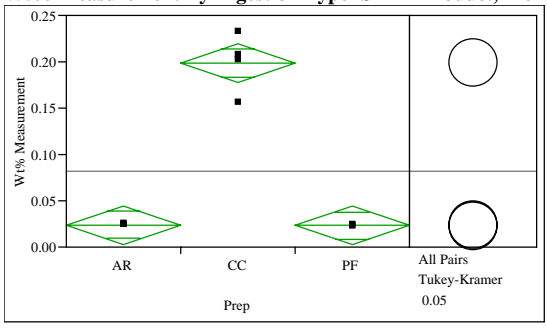

Means Comparisons

$\begin{array}{lrr}\text { Level } & \text { Mean } \\ \text { CC } & \text { A } & 0.19872387\end{array}$

AR $\quad$ A 0.02405000

PF $\quad$ B 0.02342500

Levels not connected by same letter are significantly different.

Wt $\%$ Measurement By Digestion Type=SRAT Product, Element $=\mathrm{Ca}$

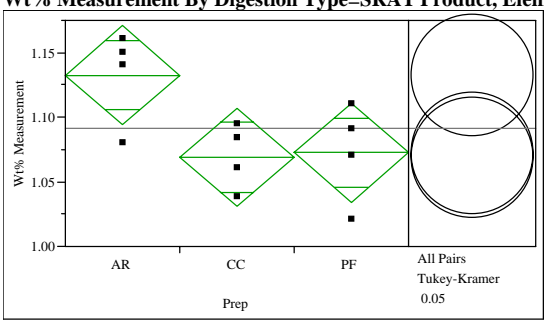

Means Comparisons

Level Mean

$\begin{array}{lll}\mathrm{AR} & \text { A } 1.1325000 \\ \mathrm{PF} & \text { A } 1.0725000\end{array}$

CC A 1.0689745

Levels not connected by same letter are significantly different.

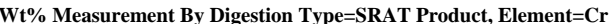

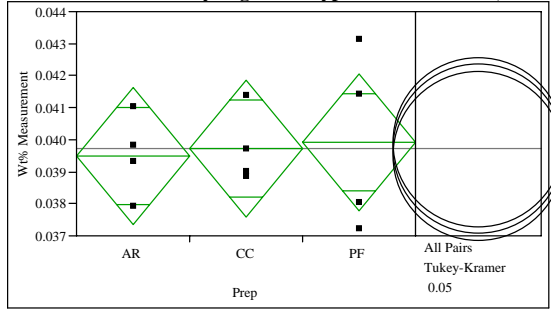

Means Comparisons

$\begin{array}{lrr}\text { Level } & \text { Mean } \\ \text { PF } & \text { A } 0.03992500\end{array}$

$\begin{array}{lll}\text { PF } & \text { A } & 0.03992500 \\ \text { CC } & \text { A } & 0.03972001\end{array}$

AR A 0.03950000

Levels not connected by same letter are significantly different.

Wt $\%$ Measurement By Digestion Type=SRAT Product, Element $=\mathrm{Cu}$

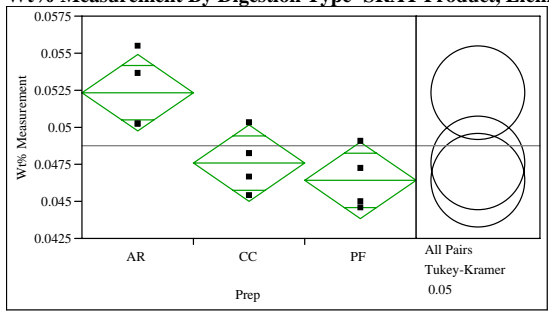

Means Comparisons

$\begin{array}{lrr}\text { Level } & & \text { Mean } \\ \text { AR } & \text { A } & 0.05235000 \\ \text { CC } & \text { B } & 0.04758194\end{array}$

PF $\quad$ B 0.04640000

Levels not connected by same letter are significantly different.

Wt\% Measurement By Digestion Type=SRAT Product, Element=Fe

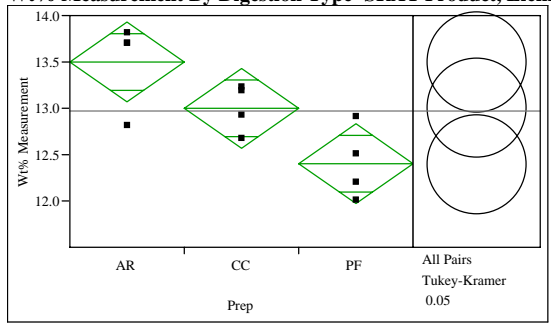

Means Comparisons

$\begin{array}{lrr}\text { Level } & \text { Mean } \\ \text { AR A } & 13.500000\end{array}$

$\begin{array}{lrll}\text { CC } & \text { A } & \text { B } & 13.000378 \\ \text { PF } & \text { B } & 12.400000\end{array}$

Levels not connected by same letter are significantly different.
Wt $\%$ Measurement By Digestion Type=SRAT Product, Element=K

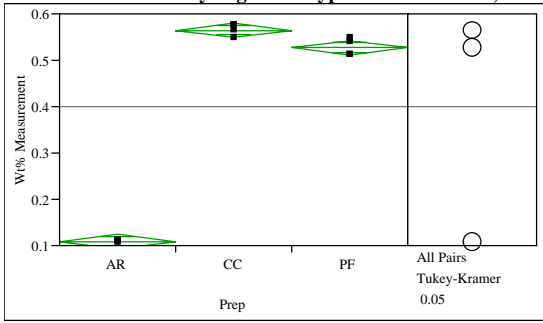

Means Comparisons

$\begin{array}{lrr}\text { Level } & \text { Mean } \\ \text { CC A } & 0.56527208 \\ \text { PF } & \text { B } & 0.52725000\end{array}$

$\begin{array}{llll}\text { PF } & \text { B } & 0.56527208 \\ \text { AR } & & 0.52725000 \\ & & \text { C } & 0.10825000\end{array}$

Levels not connected by same letter are significantly different.

Wt $\%$ Measurement By Digestion Type=SRAT Product, Element=Li

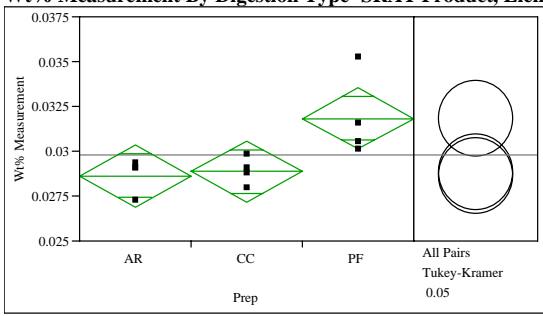

Means Comparisons

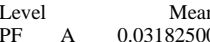

$\begin{array}{llll}\text { PF } & \text { A } & 0.03182500 \\ \text { CC } & \text { A } & \text { B } & 0.02886200\end{array}$

AR

Wt\% Measurement By Digestion Type=SRAT Product, Element $=\mathrm{Mg}$

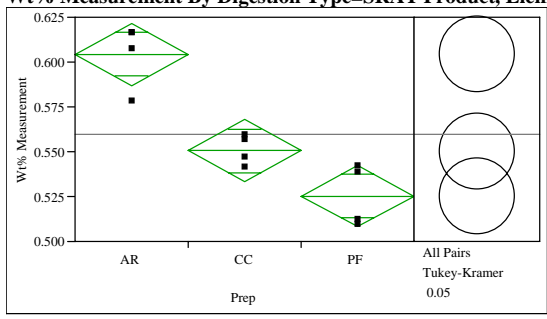

Means Comparisons

Level Mean

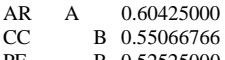

Levels not connected by same letter are significantly different. 


\section{Exhibit A1. Statistical Comparisons of Digestion Methods}

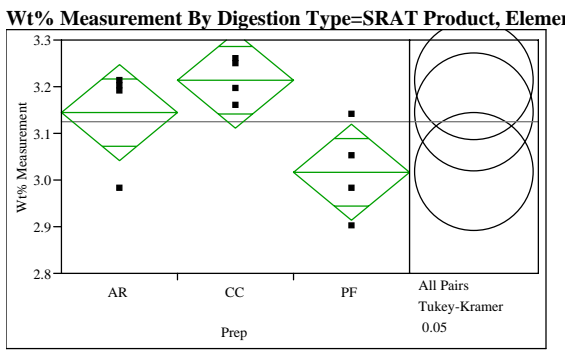

Means Comparisons

$\begin{array}{lr}\text { Level } & \text { Mean } \\ \text { CC A } 3.2141689\end{array}$

AR A B 3.1450000

PF B 3.0175000

Levels not connected by same letter are significantly different.

Oneway Analysis of Wt\% Measurement By Prep Type=SRAT Product,

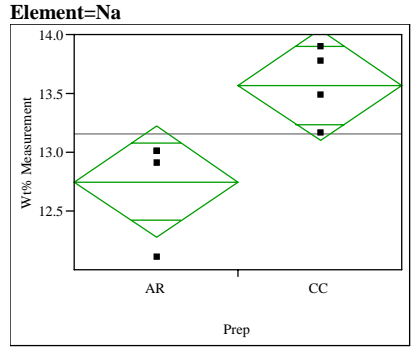

Missing Rows 4

Oneway Anova

Rsquare

$\begin{array}{ll}0.603093 \\ \text { Adj Rsquare } & 0.536942\end{array}$

Root Mean Square Error $\quad 0.384604$

Observations (or Sum Wgts)

Analysis of Variance

Source DF Sum of Squares Mean Square F Ratio Prob $>$ F $\begin{array}{lllllll}\text { Prep } & 1 & 1.3485746 & 1.34857 & 9.1169 & 0.0234\end{array}$ E. Total $7 \quad 0.085220$

Means for Oneway Anova

Level Number Mean Std Error Lower 95\% Upper 95\% $\begin{array}{lrrrrr}\mathrm{AR} & 4 & 12.7500 & 0.19230 & 12.279 & 13.221 \\ \mathrm{CC} & 4 & 13.5712 & 0.19230 & 13.101 & 14.042\end{array}$

Std Error uses a pooled estimate of error variance

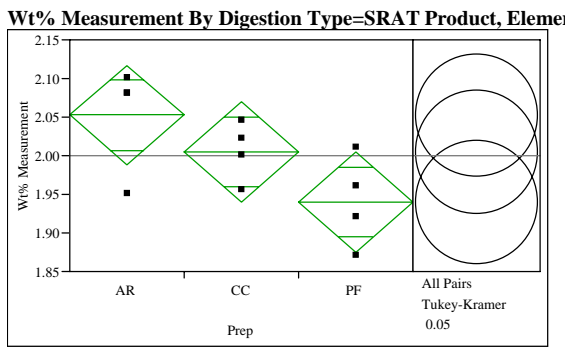

\section{Means Comparisons}

AR A 2.0525000

CC A 2.005086

Levels not connected by same letter are significantly different.

Oneway Analysis of Wt\% Measurement By Prep Type=SRAT Product,

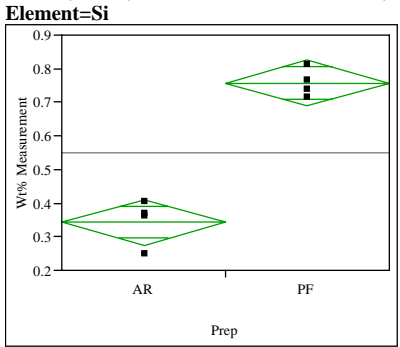

Missing Rows 4

Oneway Anova

Rsquare

0.948412

Adj Rsquare $\quad 0.939814$

Root Mean Square Error $\quad 0.055679$

Observations (or Sum Wgts) $\quad 0.55025$

Analysis of Variance

Source DF Sum of Squares Mean Square F Ratio Prob $>F$ $\begin{array}{lllllll}\text { Prep } & 1 & 0.34196450 & 0.341965 & 110.3052 & <.000\end{array}$

C. Total $\quad 7 \quad 0.36056550$

Means for Oneway Anova

Level Number Mean Std Error Lower 95\% Upper 95\% $\begin{array}{lllllll}\text { AR } & 4 & 0.343500 & 0.02784 & 0.27538 & 0.41162\end{array}$ $\begin{array}{llllll}\text { PF } & 4 & 0.757000 & 0.02784 & 0.68888 & 0.82512\end{array}$ Std Error uses a pooled estimate of error variance

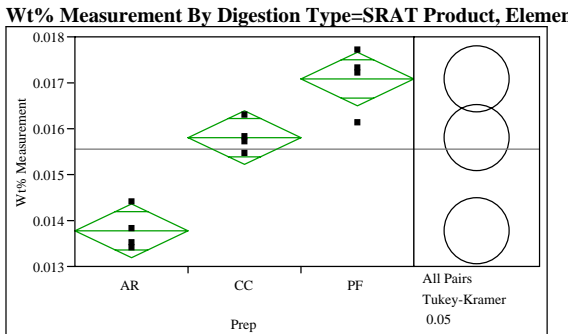

Means Comparisons

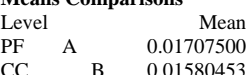

$\begin{array}{lllr}\text { CC } & \text { B } & 0.01707500 \\ \text { AR } & & 0.01580453 \\ & & \text { C } & 0.01377500\end{array}$

Levels not connected by same letter are significantly different.

Wt $\%$ Measurement By Digestion Type=SRAT Product, Element=U

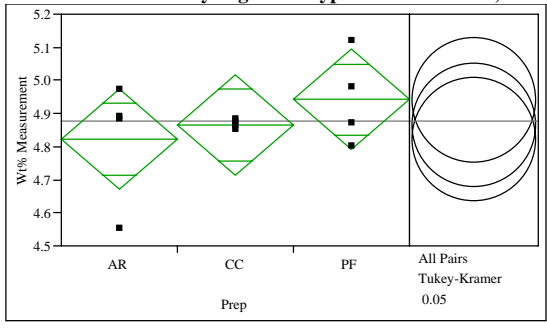

Means Comparisons

$\begin{array}{lrl}\text { Level } & \text { Mean } \\ \text { PF } & \text { A } 4.9425000\end{array}$

$\begin{array}{lll}\text { CC } & \text { A } & 4.9425000 \\ \text { AR } & \text { A } & 4.82250000 \\ \text { L }\end{array}$

AR A 4.8225000
Levels not connected by same letter are significantly different. 


\section{Exhibit A1. Statistical Comparisons of Digestion Methods}

Oneway Analysis of Wt $\%$ Measurement By Prep Type=SRAT Product,

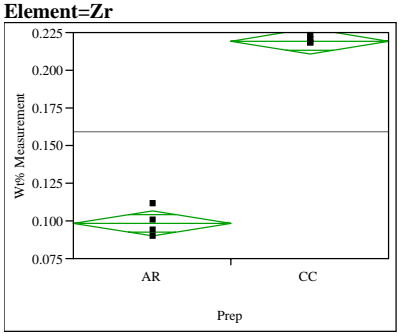

Missing Rows 4

Oneway Anova

Summary of Fit

Rsquare

0.990337

0.988727
0.006895

Mean of Respons

0.1589
8

Analysis of Variance

Source DF Sum of Squares Mean Square F Ratio Prob > F

$\begin{array}{llllll} & 1 & 0.02923362 & 0.029234 & 614.9268 & <.0001\end{array}$

$\begin{array}{lll}\text { Error } & 6 & 0.00028524 \\ \text { C. Total } & 7 & 0.0295188\end{array}$

Means for Oneway Anova

Level Number Mean Std Error Lower 95\% Upper 95\%

$\begin{array}{llllllll}\text { AR } & & 4 & 0.098450 & 0.00345 & 0.09001 & 0.10689 \\ \text { CC } & & 4 & 0.219350 & 0.00345 & 0.21091 & 0.22779\end{array}$

Std Error uses a pooled estimate of error variance

Wt $\%$ Measurement By Digestion Type=SRAT Receipt, Element=Al

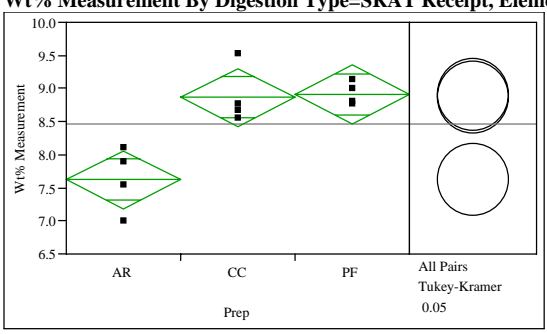

Means Comparisons

$\begin{array}{lrr}\text { Level } & \text { Mean } \\ \text { PF } & \text { A } & 8.9125000 \\ & \text { A } & 8.670000\end{array}$

$\begin{array}{lllrl}C C & \text { A } & 8.8678370 \\ \text { AR } & \text { B } & 7.6200000\end{array}$

Levels not connected by same letter are significantly different.
Wt $\%$ Measurement By Digestion Type=SRAT Receipt, Element=B

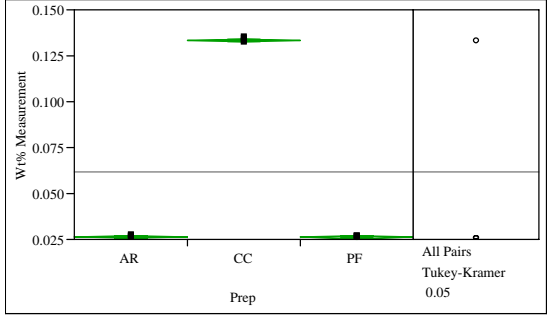

Means Comparisons

$\begin{array}{lr}\text { Means Comparisons } \\ \text { Level } & \text { Mean } \\ \text { CC A } & 0.13331590\end{array}$

AR B 0.02625000

$\mathrm{PF}$
Levels not connected by same letter are significantly different.

$\mathrm{Wt}_{\mathrm{t}} \%$ Measurement By Digestion Type=SRAT Receipt, Element=Ca

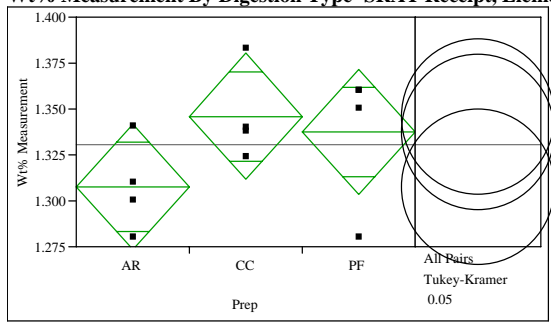

Means Comparisons

LC ${ }^{\text {L }}$ A 1.345945

PF A 1.3375000

AR A 1.3075000

列

$\mathrm{W}_{\mathrm{t}} \%$ Measurement By Digestion Type=SRAT Receipt, Element $=\mathrm{Cr}$

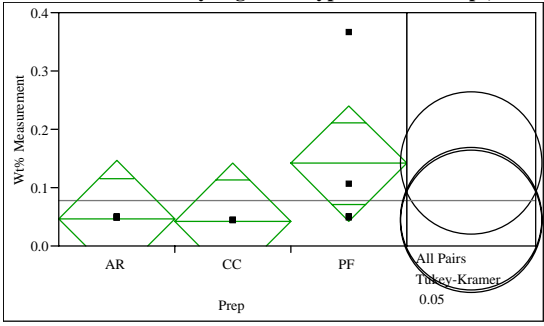

Means Comparisons

$\begin{array}{lr}\text { Level } & \text { Mean } \\ \text { PF } & \text { A } 0.14127500\end{array}$

AR A 0.04665000

CC A 0.04242121

Levels not connected by same letter are significantly different.
Wt $\%$ Measurement By Digestion Type=SRAT Receipt, Element $=\mathrm{Cu}$

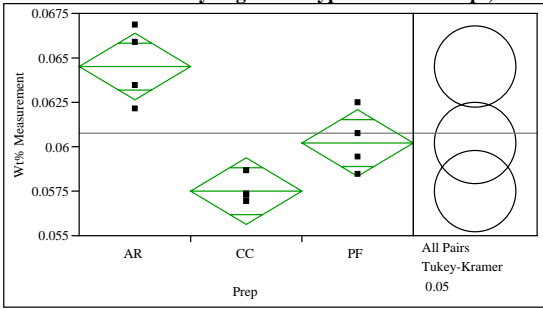

Means Comparisons

$\begin{array}{lr}\text { Level } & \text { Mean } \\ \text { AR A } & 0.06452500\end{array}$

PF $\quad$ B 0.06022500

Levels not connected by same letter are significantly different.

Wt\% Measurement By Digestion Type=SRAT Receipt, Element=Fe

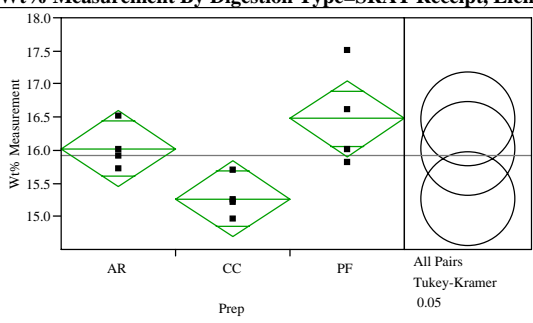

Means Comparisons

$\begin{array}{lrr}\text { Level } & \text { Mean } \\ \text { PF } & \text { A } & 16.475000\end{array}$

AR A B 16.025000

Levels not connected by same letter are significantly different.

Wt $\%$ Measurement By Digestion Type=SRAT Receipt, Element $=K$

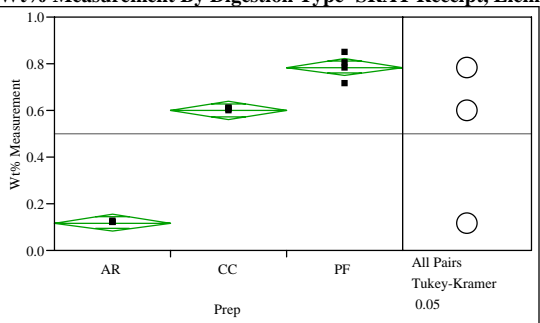

Means Comparisons

$\begin{array}{lrr}\text { Level } & \text { Mean } \\ \text { PF } & \text { A } & 0.78475000\end{array}$

$\begin{array}{lllll} & \text { PF } & & & 0.78475000 \\ \text { CC } & & \text { B } & 0.60043039 \\ \text { AR } & & & \text { C } & 0.11800000\end{array}$

AR 


\section{Exhibit A1. Statistical Comparisons of Digestion Methods}

$W_{t} \%$ Measurement By Digestion Type=SRAT Receipt, Element $=\mathrm{L}$

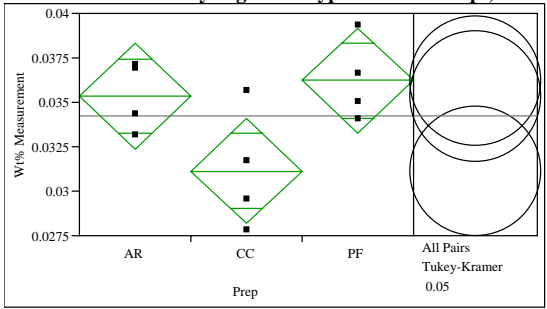

Means Comparisons

$\begin{array}{lrr}\text { Level } & \text { Mean } \\ \text { PF } & \text { A } 0.03622500\end{array}$

AR A 0.03535000

Levels not connected by same letter are significantly different.

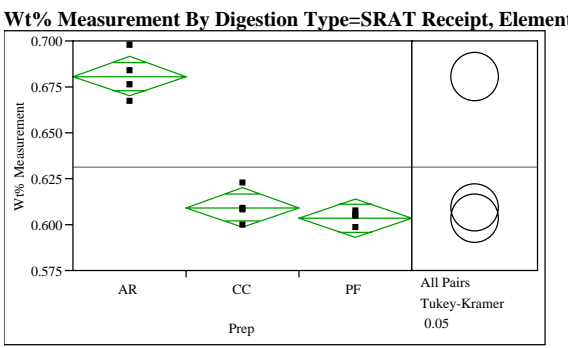

Means Comparisons

Level Mean

$\begin{array}{lll}\text { AR } & \text { A } & 0.68075000 \\ \text { CC } & \text { B } & 0.60935539 \\ \text { PF } & \text { B } & 0.60350000\end{array}$

Levels not connected by same letter are significantly different.

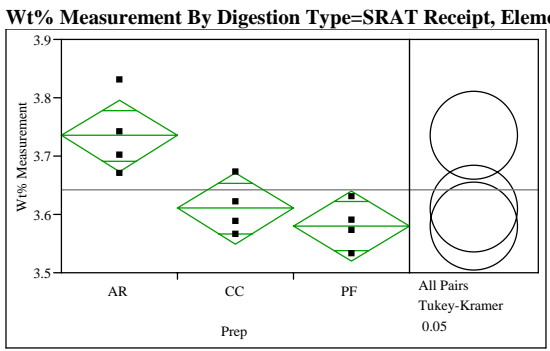

Means Comparisons

$\begin{array}{lrr}\text { Level } & \text { Mean } \\ \text { AR A } & 3.7350000\end{array}$

CC $\quad$ B 3.6102929

Levels not connected by same letter are significantly different.
Oneway Analysis of Wt $\%$ Measurement By Prep Type=SRAT Receipt,

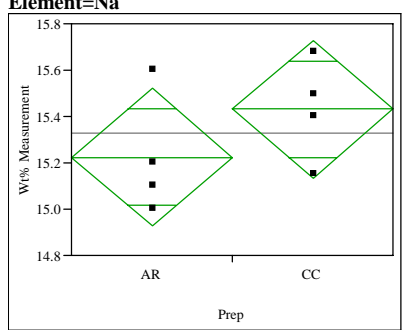

Missing Rows 4

Oneway Anova
Summary of Fit

Rsere

Adj Rsquare

Mean of Response 1532801

Observations (or Sum Wgts) 8

Analysis of Variance

Source DF Sum of Squares Mean Square F Ratio Prob $>F$ $\begin{array}{llllll}\text { Prep } & 1 & 0.0848260 & 0.084893 & 1.4470 & 0.2743\end{array}$

$\begin{array}{lll}\text { C. Total } & 7 & 0.43690953\end{array}$

Means for Oneway Anov

Level Number Mean Std Error Lower 95\% Upper 95\% $\begin{array}{lllllll}\text { AR } & 4 & 15.2250 & 0.12111 & 14.929 & 15.521 \\ \text { CR } & 4 & 15.4310 & 0.12111 & 15.135 & 15.727\end{array}$

Std Error uses a pooled estimate of error variance

Wt\% Measurement By Digestion Type=SRAT Receipt, Element $=\mathbf{N i}$

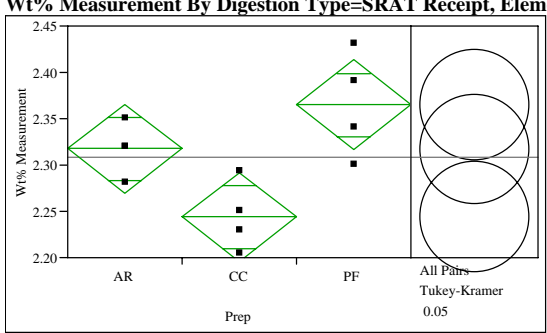

Means Comparisons

$\begin{array}{lr}\text { Level } & \text { Mean } \\ \text { PF A } & 2.3650000\end{array}$

AR A $\quad$ A 2.3175000

Levels not connected by same letter are significantly different.
Oneway Analysis of Wt\% Measurement By Prep Type=SRAT Receipt,

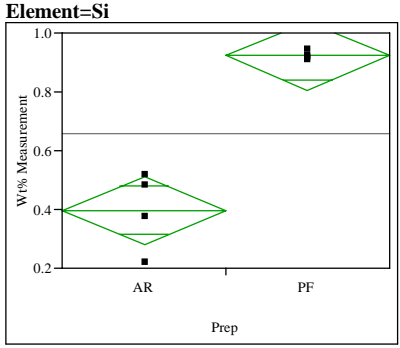

Missing Rows 4

Oneway Anova
Summary of Fit

Rsquare

\begin{tabular}{ll} 
Rsquare & 0.910578 \\
Ajj Rsquare & 0.895674 \\
Root Mean Square Error & 0.095123 \\
\hline
\end{tabular}

Mean of Response $\quad 0.659625$

Source DF Sum of Squares Mean Square F Ratio Prob $>F$ $\begin{array}{lllllll}\text { Prep } & 1 & 0.55282613 & 0.552826 & 61.0973 & 0.0002\end{array}$ C. Total $7 \quad 0.6071158$

Means for Oneway Anova

Level Number Mean Std Error Lower 95\% Upper 95\%

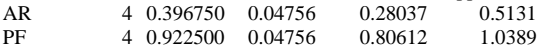

Std Error uses a pooled estimate of error variance

Wt $\%$ Measurement By Digestion Type=SRAT Receipt, Element=Ti

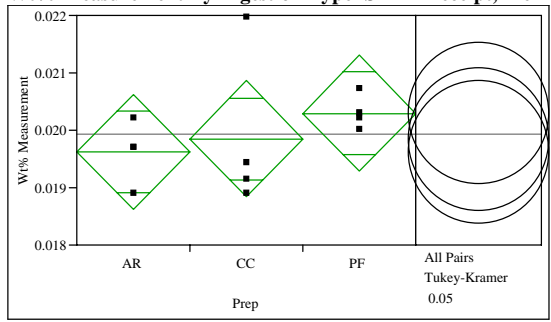

Means Comparisons

PF A 0.02030000

CC A 0.01985123

AR A 0.01962500

A 


\section{Exhibit A1. Statistical Comparisons of Digestion Methods}

Wt $\%$ Measurement By Digestion Type=SRAT Receipt, Element $=U$

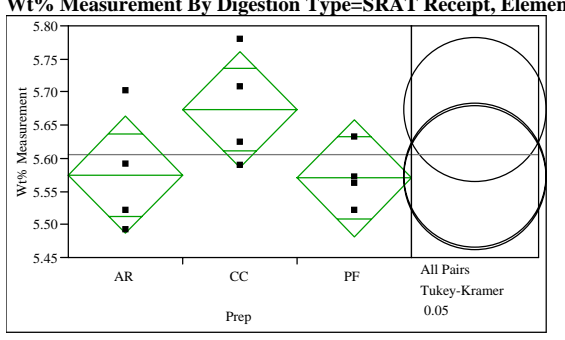

Means Comparisons

$\begin{array}{lll}\text { Level } & \text { Mean } \\ \text { CC } 5.6736862 & \end{array}$

AR A 5.5750000

PF A 5.5700000

ls not connected by same letter are significantly different.

Oneway Analysis of Wt\% Measurement By Prep Type=SRAT Receipt,

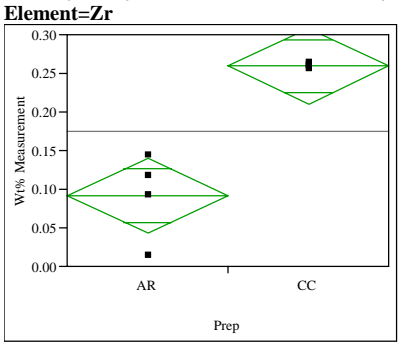

Missing Rows 4

Summary of Fit

Rsquare

Adj Rsquare

Nen $\quad 0.040037$

Observations (or Sum Wgts) 8

Analysis of Variance

Source DF Sum of Squares Mean Square F Ratio Prob $>$ F

$\begin{array}{llllll}\text { Prep } & 1 & 0.05622981 & 0.056230 & 35.0784 & 0.0010\end{array}$

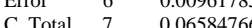

Means for Oneway Anova

$\begin{array}{lrrrrr}\text { Level } & \text { Number } & \text { Mean } & \text { Std Error } & \text { Lower 95\% } & \text { Upper 95\% } \\ \text { AR } & 4 & 0.091600 & 0.02002 & 0.04262 & 0.14058\end{array}$

$\begin{array}{llllll}\text { CC } & 4 & 0.259275 & 0.02002 & 0.21029 & 0.30826\end{array}$ 
$\underline{\text { Distribution }}$

C.J. Bannochie, 773-42A

M.J. Barnes, 773-A

N.E. Bibler, 773-A

J.M. Bricker, 704-27S

L.M. Chandler, 773-A

C.J. Coleman, 773-A

B.A. Davis, 773-A

T.B. Edwards, 773-42A

M.T. Feller, 704-28S

T.L. Fellinger, 704-26S

J.C. Griffin, 773-A

C.C. Herman, 999-W

J.F. Iaukea, 704-30S

R.T. McNew, 704-27S

R.N. Mahannah, 704-28S

T.A. Nance, 773-42A

A.B. Osteen, 704-28S

J.E. Occhipinti, 704-S

D.K. Peeler, 999-W

F.M. Pennebaker, 773-A

J.W. Ray, 704-S

M.E. Stone, 999-W

ADS Files 